TITLE:

\title{
Manufacturability evaluation for molded parts using fictitious physical models, and its application in topology optimization
}

\section{$\operatorname{AUTHOR}(\mathrm{S}):$}

Sato, Yuki; Yamada, Takayuki; Izui, Kazuhiro; Nishiwaki, Shinji

\section{CITATION:}

Sato, Yuki ... [et al]. Manufacturability evaluation for molded parts using fictitious physical models, and its application in topology optimization. The International Journal of Advanced Manufacturing Technology 2017, 92(1-4): 1391-1409

\section{ISSUE DATE:}

2017-09

URL:

http://hdl.handle.net/2433/226740

\section{RIGHT:}

The final publication is available at Springer via http://dx.doi.org/10.1007/s00170-017-0218-0.; The full-text file will be made open to the public on 01 September 2018 in accordance with publisher's 'Terms and Conditions for SelfArchiving'; この論文は出版社版でありません。引用の際には出版社版をご確認ご利用ください。; This is not the published version. Please cite only the published version. 


\title{
Manufacturability evaluation for molded parts using fictitious physical models, and its application in topology optimization
}

\author{
Yuki Sato · Takayuki Yamada · Kazuhiro Izui · Shinji Nishiwaki
}

Received: date / Accepted: date

\begin{abstract}
Manufacturing methods using molds, such as casting and injection molding, are widely used in industries. A basic requirement when using such manufacturing methods is that design engineers must design products so that they incorporate certain geometrical features that allow the mold parts to be removed from the created solid object. In the present study, we propose a manufacturability evaluation method especially adapted for the use of molds. To evaluate the manufacturability, we introduce fictitious physical models that are described by steady-state anisotropic advectiondiffusion equations. In these fictitious physical models, material domains have a virtual source term and the advection directions are aligned with the directions along which the mold parts are parted. Void regions, where the values of all fictitious physical fields are high, then represent either undercut geometries that would prevent the mold from being released, or interior voids that cannot be cast. Consequently, manufacturability can be evaluated using these fictitious physical fields. Furthermore, in the present study, we integrate this evaluation method with topology optimization and propose a scheme for imposing a molding constraint within the topology optimization procedure. This newly proposed topology optimization method can consider the position of mold parting lines prior to the detailed optimization procedure. Several numerical examples are provided to demonstrate the validity and effectiveness of the proposed method.
\end{abstract}

Keywords Manufacturability · Fictitious physical model · Topology optimization · Geometrical constraint · Molding constraint

\section{Y. Sato $\cdot$ T. Yamada $\cdot$ K. Izui $\cdot$ S. Nishiwaki}

Department of Mechanical Engineering and Science, Kyoto University,

C3, Kyotodaigaku-katsura, Nishikyo-ku, Kyoto 615-8540, Japan

Tel.: +81-75-383-3602

Fax: +81-75-383-3601

E-mail: satou.yuuki.87x@st.kyoto-u.ac.jp

\section{Introduction}

Manufacturing methods exploiting the use of molds in casting or injection processes are widespread in many industries. The most important manufacturing requirements when using a molding process is to ensure that the shape of the product has certain geometrical features that allow the mold parts to be removed, while maximizing the performances of the product under consideration [1], a design process that usually requires much trial and error and relies on the experience of design engineers. In such scenarios, the design for manufacturing (DFM) process [2] is often used. The DFM concept aims to obtain product designs that are easy to manufacture and some studies incorporate CAE analysis for a casting process as a DFM tool [3, 4]. In DFM, it is important to consider the performance of products together with their manufacturing costs and manufacturability. However, conventional DFM typically focuses on a simple method for evaluating manufacturing costs $[2,5,6]$ via, for example, simplification and standardization of design and manufacturing processes [7], although the lowering of performances due to such simplification has recently been considered in some research [8, 9]. Research on DFM methods for improving product designs while ensuring their performance is relatively scarce, and reliance on design engineers' expertise to achieve product design improvements that also maintain or improve performance is still the norm. To ameliorate this situation, a new design support system that can consider product performances together with their manufacturing costs and manufacturability in the early design phase is required [10].

In the present study, we therefore propose a manufacturability evaluation method that can be incorporated in structural optimization process as a DFM tool. To evaluate manufacturability, we introduce fictitious physical models that are described by steady-state anisotropic advection-diffusion equa- 
tions. In these fictitious physical models, virtual heat generation occurs in material domains and the advection directions are aligned with the direction along which the mold parts are parted. Void regions, where the values (i.e. the fictitious temperatures) of all fictitious physical fields are high, then represent either undercut geometries that would prevent the mold from being released, or interior voids that cannot be cast. In this manner, manufacturability can be evaluated using the fictitious physical fields.

Furthermore, by incorporating the proposed method in a structural optimization method, well-known as a powerful tool for deriving optimal structures based on mathematical and physical principles, it can be applied within the structural design process. Structural optimization can be classified into three types: sizing [11, 12], shape [13, 14], and topology optimization $[15,16]$. Among these, topology optimization offers the greatest potential for exploring highperformance structures, and has been applied in various complex problems in the literature [17-19]. The field of topology optimization for structural problems is essentially mature, and the results of topology optimization approaches are effectively used in many industries, such as automobile and aeronautical industries. In particular, the solid isotropic material with penalization (SIMP) method [20] is particularly popular in the topology optimization field. Level set-based shape optimization methods [21-23], which essentially exclude grayscale areas that may appear in the optimal configurations obtained by SIMP methods, are another popular approach in structural optimization.

Among these structural optimization methods, the objective of topology optimization in most previous research has mainly focused on deriving optimal structures for certain devices to improve their performance. As a result, when topology optimization solutions are applied in practical manufacturing circumstances, design engineers often must interpret and modify the results to satisfy manufacturability conditions [24]. However, maintaining the performance of optimal configurations obtained during subsequent shape modification processes is often difficult for design engineers. Consequently, the performance of modified design candidates is often far below that of the originally obtained optimal solutions. This difficulty may make the use of topology optimization in real world engineering a challenging task. To overcome this problem, it is important to consider manufacturing requirements during the optimization process. How to implement manufacturing constraints, i.e., constraints due to particular manufacturing requirements, is now one of the most interesting topics in the field of topology optimization.

Manufacturing constraints are types of geometrical constraints that ensure that optimal configurations have certain desired geometrical features that permit their manufacture using certain processes. Researchers have proposed a variety of methods for imposing geometrical constraints in shape and topology optimization [25]. Length scale constraints are one of the most studied geometrical constraints in topology optimization. Imposing a minimum length scale constraint prevents the appearance in optimal configurations of excessively complex substructures and hinges that would be difficult to manufacture. Several minimum length scale geometrical constraint methods have been proposed for use in SIMP schemes [26-28].

On the other hand, maximum length scale constraints are also important in certain manufacturing processes. In die casting, for instance, a maximum length scale constraint can suppress the appearance of void shrinkage cavities that decrease fatigue strength, which may occur in large structural members. A consequence of imposing a maximum length scale constraint can also be exploited as a structural redundancy measurement, whereby loads are redistributed and load paths diversified by replacing each large structural member with a number of smaller features [29]. Several methods for imposing maximum length scale constraints have been proposed $[29,30]$

Length scale control methods that can impose both minimum and maximum length scale constraints have also been proposed [31,32] using a level set-based shape optimization scheme. Other schemes for imposing certain manufacturing constraints in shape and topology optimizations have also been proposed. For additive manufacturing (AM), Brackett et al. [33] summarized the issues for the application of topology optimization in AM. Leary et al. [34] proposed a method in which the obtained optimal configurations are modified to satisfy an overhang constraint required to avoid the use of support material in AM, and additional schemes for imposing overhang constraints during the optimization procedure have been proposed [35, 36]. Li et al. [37] proposed a connectivity constraint to avoid enclosed voids in structures by introducing a virtual scalar field.

As for molding constraints, Zhou et al. proposed a mathematical formulation of a molding constraint [38] in a SIMP scheme. Xia et al. proposed a molding constraint method [1] in a level set-based shape optimization scheme by limiting the advection velocity in the Hamilton-Jacobi equation, a method later extended by Allaire et al. [39]. Additionally, uniform cross-section surface constraints, also called extrusion constraints, have been proposed to simplify certain designs and promote manufacturability [39-41]. A crosssection constraint can be a sufficient, but not necessary, condition for manufacturability in casting processes.

In the present study, we incorporate the proposed molding manufacturability evaluation method in a topology optimization procedure to obtain optimal configurations for certain devices that provide high performance while ensuring manufacturability using molding techniques. Here, molding manufacturability is evaluated according to fictitious physi- 

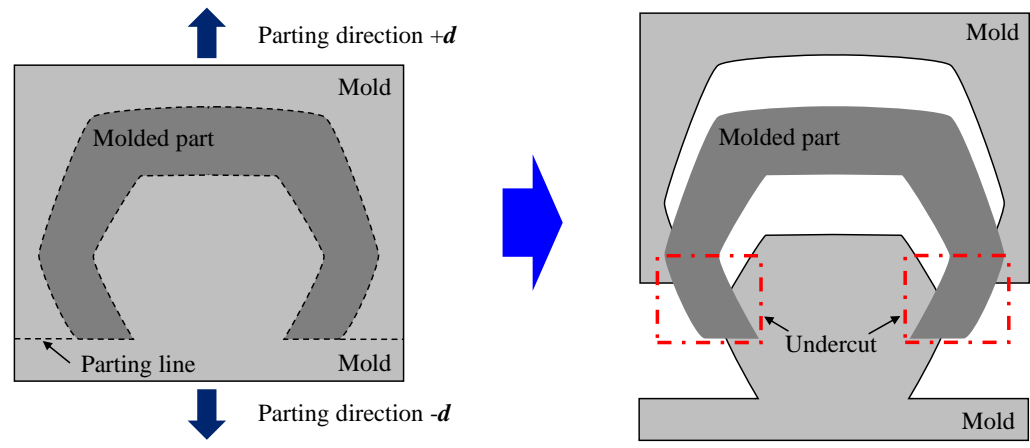

Fig. 1 Undercut geometry.

cal fields, with the molding constraint imposed in the fictitious physical models.

The remainder of this paper is organized as follows. First, we introduce the methodology for evaluating manufacturability when using molds via fictitious physical models that are governed by advection-diffusion equations. Next, we briefly discuss topology optimization methods and formulate a molding constraint within a topology optimization by applying the evaluation method. Based on this formulation, we define optimization problems that not only maximize the performance of a particular device but also ensure its manufacturability. We then construct an optimization algorithm for the topology optimization using the FEM. Finally, we provide two- and three-dimensional numerical examples to confirm the applicability of the proposed method.

\section{Evaluation of mold manufacturability via fictitious physical models}

2.1 Clarification of requirements

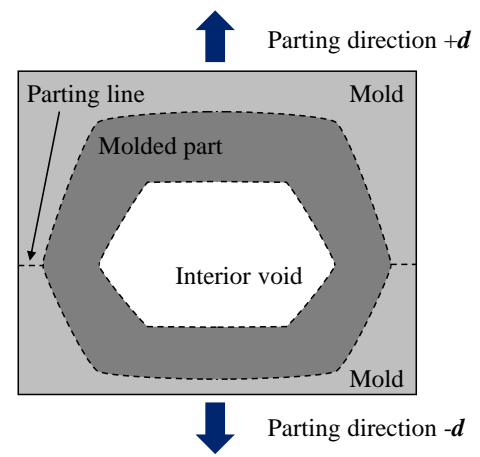

Fig. 2 Geometry with an interior void.

First, we clarify the geometrical feature requirements for manufacturability when using molding techniques. Structural features that would be problematic when using cast- ing or injection molding can be classified into two types: (1) undercut geometry that would prevent the mold from being disassembled; and (2) the presence of one or more interior voids within the structure, which would make manufacturing impractical. Figure 1 shows an example of undercut geometry, where the lower mold section is captured by an undercut, preventing the mold parts from being separated at the given parting line in the given parting direction. Figure 2 shows an example of geometry with an interior void, an empty region that is completely contained within the cast material. Manufacturing structures that include an interior void by casting or injection molding is extremely cumbersome. On the other hand, a structure free from both undercuts and interior voids can be manufactured using a conventional mold, as shown in Fig. 3. The direction in which a mold is disassembled is called the parting direction, denoted as $+\boldsymbol{d}$ and $-\boldsymbol{d}$ in these figures, and the line on which two parts of a mold make contact is called the parting line. When a product will be manufactured using casting or injection molding, design engineers must ensure that undercuts and interior voids are avoided.

\subsection{Formulation for a process using two-part molds}

\subsubsection{Introduction of fictitious physical models}

When a designed component has a simple structure, it is easy to determine whether or not it can be manufactured using a molding technique. However, when a design engineer designs a component that has a complex structure, it may be difficult to evaluate such manufacturability. In particular, when using structural optimization methods to derive optimal structures, design engineers inevitably must interpret and modify the initially obtained results, to satisfy manufacturability requirements. Consequently, a trial and error process is required and design engineers must have broad experience when designing sophisticated components that will be manufactured by casting or injection molding. To overcome these issues, we propose a molding manufacturability eval- 

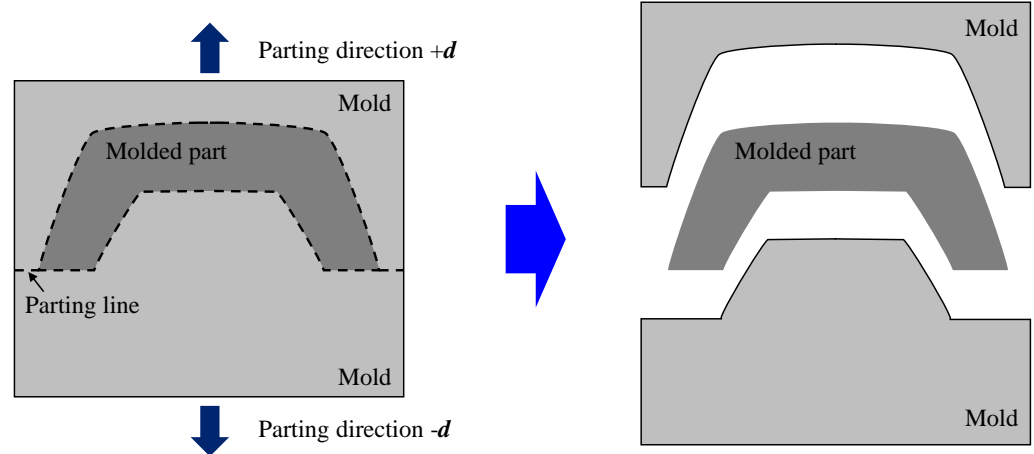

Fig. 3 Manufacturable geometry for casting or injection molding processes.

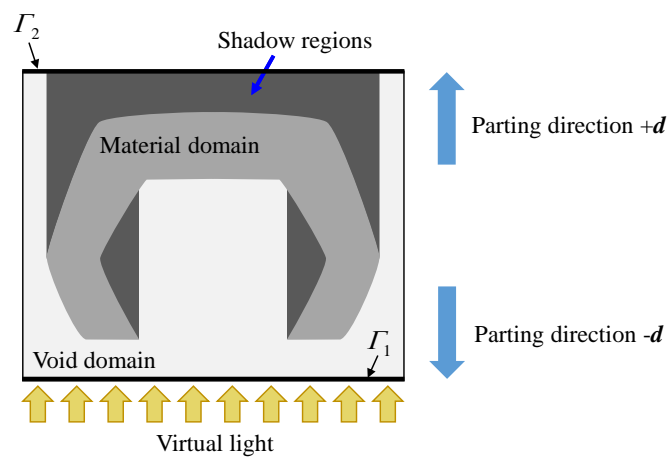

(a)

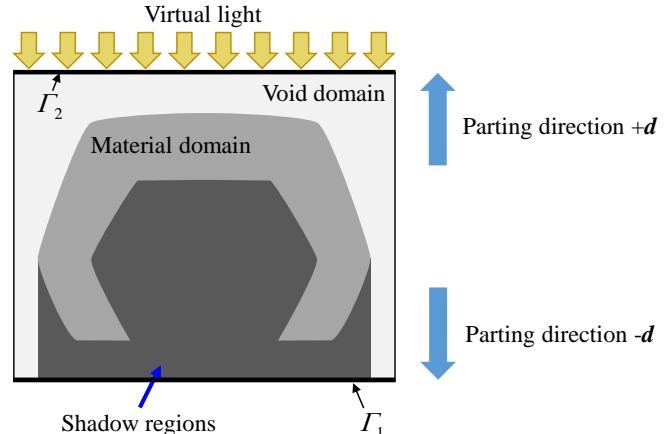

(b)

Fig. 4 Virtual light shined on a structure: (a) From bottom to top; (b) From top to bottom.

uation method using fictitious physical models to implicitly evaluate geometrical features.

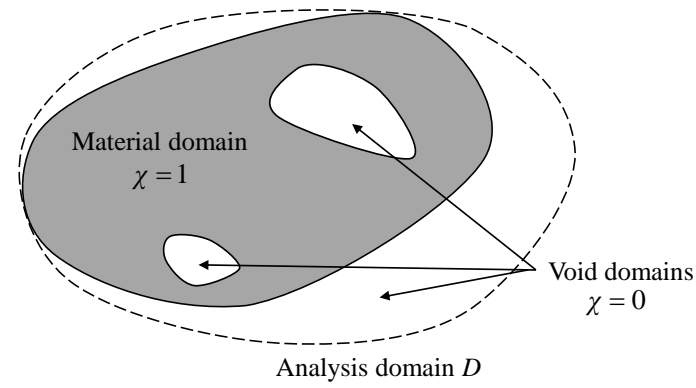

Fig. 5 Characteristic function.

Consider two cases, one in which light is shined upon a structure from a line $\Gamma_{1}$ at the bottom toward line $\Gamma_{2}$ at the top, and the other in which light travels from top line $\Gamma_{2}$ to bottom line $\Gamma_{1}$, both along the parting direction, as shown in Fig. 4. Undercut and interior void regions can then be described as regions that receive no light, shadow regions in both cases. Analogously, we consider virtual heat flows that are only conducted along parting directions. That is, we in- troduce two fictitious physical models that are mathematically described with advection-diffusion equations, using opposite advection directions. Let $\psi_{1}$ and $\psi_{2}$ denote the state variables, and let $\boldsymbol{A}$ and $\boldsymbol{V}$ denote the non-dimensional second order diffusion tensor and the non-dimensional advection vector, respectively. We introduce two fictitious physical models, described as

$$
\begin{array}{ll}
-L^{2} \operatorname{div}\left(\boldsymbol{A} \nabla \psi_{1}\right)+L \boldsymbol{V} \cdot \nabla \psi_{1}=\beta \chi\left(1-\psi_{1}\right) & \text { in } D \\
\psi_{1}=0 & \text { on } \Gamma_{1} \\
\boldsymbol{n} \cdot \nabla \psi_{1}=0 & \text { on } \partial D \backslash \Gamma_{1}
\end{array}
$$

$-L^{2} \operatorname{div}\left(\boldsymbol{A} \nabla \psi_{2}\right)-L \boldsymbol{V} \cdot \nabla \psi_{2}=\beta \chi\left(1-\psi_{2}\right) \quad$ in $D$

$\psi_{2}=0$,

on $\Gamma_{2}$,

$\boldsymbol{n} \cdot \nabla \psi_{2}=0$

on $\partial D \backslash \Gamma_{1}$

where $\beta$ is a parameter, $\boldsymbol{n}$ is the outward unit vector normal to $\partial D$, and $L$ is the length of the analysis domain along the parting direction for non-dimensionalization. $\chi$ is the characteristic function defined in the analysis domain $D$ and it has a value of 1 in the material domain and 0 in void domains, as shown Fig. 5. 
In this model, $\boldsymbol{A}$ is anisotropic and has larger values in the parting direction than those in the orthogonal direction, and $\boldsymbol{V}$ is a vector aligned with the $\boldsymbol{d}$ direction. If we set $\beta$ and the norm of $\boldsymbol{V}$ to sufficiently large values, $\psi_{1}$ approaches 1 in the material domain and shadow regions when viewed from $\Gamma_{1}$, and $\psi_{2}$ approaches 1 in material domain and shadow regions when viewed from $\Gamma_{2}$. Consequently, the void regions where the values of both fictitious physical fields, $\psi_{1}$ and $\psi_{2}$, are high correspond to shadow regions and represent either undercuts or interior void regions. Therefore, the presence of undercut and interior void regions can be evaluated by visualizing the shadow regions using the term $\psi_{1} \psi_{2}(1-\chi)$ that represents the intersection of the shadow regions. The validity of these fictitious physical models is confirmed in Section 5. The proposed method solves physical models described with partial differential equations, similar to the method used in existing CAE tools, so the proposed method is highly compatible.
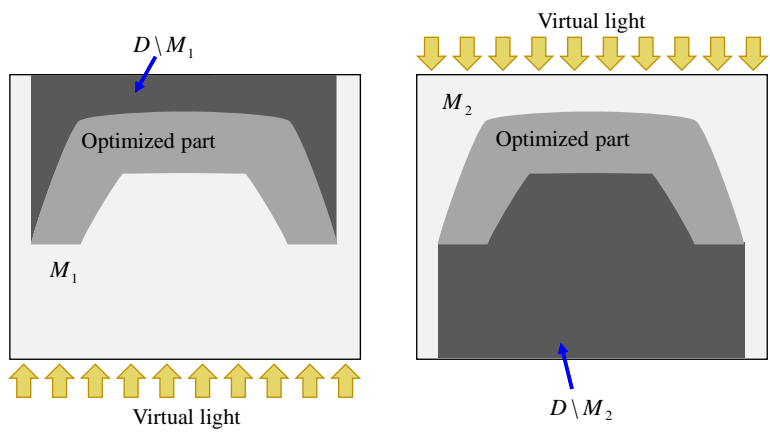

Fig. 6 Mold design using fictitious physical models.

\subsubsection{Application to structural optimization}

We can easily incorporate the molding manufacturability evaluation method described above with a structural optimization to obtain optimal structures for certain product parts or components that provide high performance while ensuring manufacturability based on the use of molds. Consider a structural optimization problem to determine an optimal configuration of a domain filled with material in order to minimize the value of an objective functional, $F$. Let $\Omega$ and $\Gamma$ denote the material domain and its boundary, respectively. Denote by $f_{\mathrm{d}}$ and $f_{\mathrm{b}}$ the integrands of the objective functional defined in the domain $\Omega$ and on boundary $\Gamma$, respectively. A position vector in $\Omega$ and its state variable vector are denoted as $\boldsymbol{x}$ and $\boldsymbol{u}$, respectively. A structural optimization problem is then formulated as

$\inf _{\Omega} \quad F=\int_{\Omega} f_{\mathrm{d}}(\boldsymbol{x}, \boldsymbol{u}, \nabla \boldsymbol{u}) d \Omega+\int_{\Gamma} f_{\mathrm{b}}(\boldsymbol{x}, \boldsymbol{u}, \nabla \boldsymbol{u}) d \Gamma$.
On the other hand, undercut and interior void regions can be nullified in the structural optimization procedure by minimizing the following functional:

$F_{\mathrm{M}}=\int_{D} \psi_{1} \psi_{2}(1-\chi) d \Omega$.

That is, by minimizing $F_{\mathrm{M}}$ together with objective functional $F$, which aims to improve a physical performance of a certain device, the molding constraint is incorporated in the structural optimization.

\subsubsection{Application to mold design}

Furthermore, once the optimization procedure is finished, appropriate mold designs for manufacturing the structurally optimized product components can be created using the fictitious physical models. Let domains $M_{1}$ and $M_{2}$ be respectively defined as follows:

$M_{1}=\left\{\forall \boldsymbol{x} \in D: \psi_{1}<c\right\}$

$M_{2}=\left\{\forall \boldsymbol{x} \in D: \psi_{2}<c\right\}$,

where $c \in(0,1)$ is a real constant. $M_{1}$ represents the regions in the model that are illuminated from $\Gamma_{1}$ and $M_{2}$ represents regions illuminated from $\Gamma_{2}$, as shown in Fig. 6. That is, $M_{1}$ and $M_{2}$ respectively represent the domain of each mold. If a domain $M_{1} \cap M_{2}$ exists, it can be included in either half of the mold in this example. We note that the optimal locations of parting lines and gates should also be considered for casting processes. However, for simplicity, the focus of the present study is to obtain an optimal configuration that provides high performance together with suitable geometrical manufacturability at the conceptual design stage.

\subsection{Formulation for process using molds with multiple parts}

The above evaluation method can easily be generalized to a process using molds consisting of multiple parts. Figure 7(a) shows the case where a three-part mold is proposed for use in a manufacturing process for a particular product shape. However, the upper part (Mold part 1) and the lower part (Mold part 2) cannot be separated in the given parting direction, since there is an undercut at the right side of the molded part. To evaluate the geometrical features here, consider cases in which virtual light illuminated the structure from multiple directions, each of which corresponds to the parting direction of a particular mold part. Undercut and interior void regions can then be described as regions that are shadow regions in all cases, as shown in Fig. 7(b). In the same manner as that used in the two-part mold case (Section 2.2), we introduce multiple fictitious physical models that are mathematically described using the advection-diffusion 


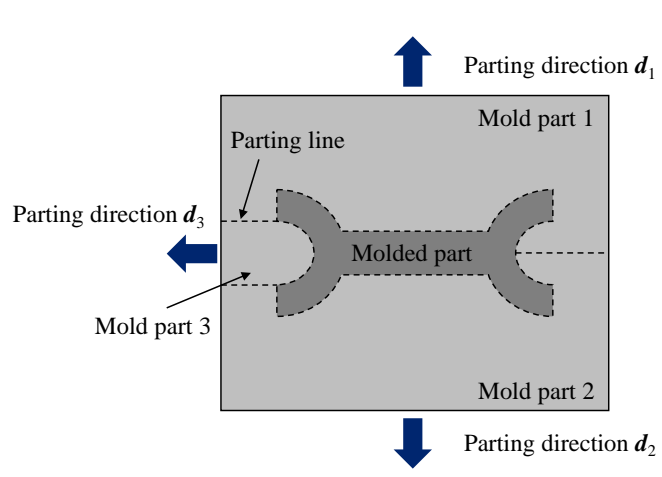

(a)

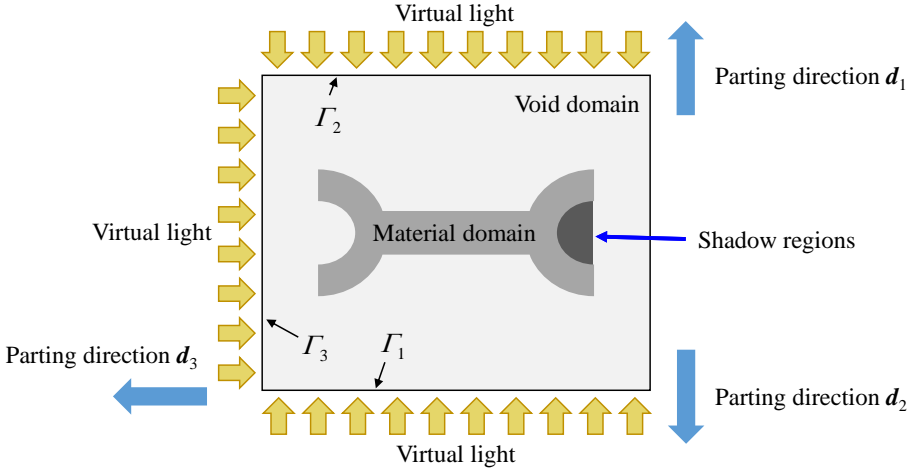

(b)

Fig. 7 Generalization to mult-part molds: (a) Three-part mold; (b) Virtual light shined on structure.

equation, each of which corresponds to the parting direction for each mold part.

First, we assume that $m$ represents the number of mold parts. Let $\psi_{i}, \boldsymbol{A}_{i}$ and $\boldsymbol{V}_{i}(i=1,2, \ldots, m)$ denote the state variables, the non-dimensional second order diffusion tensor, and the non-dimensional advection vector, respectively. We introduce fictitious physical models described as

$$
-L_{i}^{2} \operatorname{div}\left(\boldsymbol{A}_{i} \nabla \psi_{i}\right)+L_{i} \boldsymbol{V}_{i} \cdot \nabla \psi_{i}=\beta \chi\left(1-\psi_{i}\right) \quad \text { in } D
$$

$\psi_{i}=0$,

$\boldsymbol{n} \cdot \nabla \psi_{i}=0$ on $\partial D \backslash \Gamma_{i}$

for all indices $i=1,2, \ldots, m$, where

$\Gamma_{i}=\left\{\forall \boldsymbol{x} \in \partial D: \boldsymbol{d}_{i} \cdot \boldsymbol{n}>0\right\}$,

with $\partial D$ representing the boundary of $D$ and $\boldsymbol{d}_{i}$ the parting direction of $i$ th mold part. $L_{i}(i=1,2, \ldots, m)$ is the length of the analysis domain $D$ along each parting direction for nondimensionalization. In this model, $\boldsymbol{A}_{i}$ is anisotropic, with larger values in each parting direction than those in the orthogonal direction, and $\boldsymbol{V}_{i}$ is a vector in the direction opposite that of each parting direction. In the same manner as for the two-part mold case, undercut and interior void regions can then be evaluated by the term $(1-\chi) \prod_{i=1}^{m} \psi_{i}$. That is, the case of the two-part mold described in Section 2.2 is a special case where $\boldsymbol{A}_{1}=\boldsymbol{A}_{2}$ and $\boldsymbol{V}_{1}=-\boldsymbol{V}_{2}$.

The molding constraint for a mold with more than two parts can also be imposed in the structural optimization procedure in the same manner as that described in Section 2.2.2, by minimizing the following functional:

$F_{\mathrm{M}}^{m}=\int_{D}(1-\chi) \prod_{i=1}^{m} \psi_{i} d \Omega$.

By minimizing $F_{\mathrm{M}}^{m}$ together with the objective functional $F$, a molding constraint for a mold with three or more parts can be incorporated into the structural optimization.
We can also design molds with three or more parts in the same manner as that described in Section 2.2.3. Let the domain $M_{i}$ be defined as

$M_{i}=\left\{\forall \boldsymbol{x} \in D: \psi_{i}<c\right\}$,

for all indices $i=1,2, \ldots, m$ where $c \in(0,1)$ is a real constant. $M_{i}$ then represents domain filled with the $i$ th mold part. If there is an intersection of multiple domains, it can be incorporated into any convenient mold part.

\subsection{Predetermination of parting lines in the structural optimization}

In the above method for structural optimization, the parting lines are determined according to the mold design after the optimization procedure. However, in practical engineering, it is often attractive to be able to predetermine parting lines, based on the cost of fabricating the mold. The proposed method can be extended to allow predetermination of mold parting lines in a structural optimization.

Predetermining mold parting lines is comparable to predetermining certain parts of the analysis domain that is included in a certain mold part. Let $M_{i}^{\text {pre }}$ for $i=1,2, \ldots, m$ denote a sub-domain of the analysis domain that is predetermined to be filled with the $i$ th mold part, and let $D^{\text {und }}$ represent a sub-domain of the analysis domain for which the particular mold part it is to be included in is undetermined, as shown in Fig. 8. In this case, shadow regions in $M_{i}^{\text {pre }}$ viewed from $\Gamma_{i}$ represent either undercuts or interior voids, since $M_{i}^{\text {pre }}$ should be included in the $i$ th mold part. Consequently, the molding constraint for this case can be imposed in the structural optimization procedure by minimizing the following functional:

$$
F_{\mathrm{M}}^{\mathrm{pre}}=\sum_{i=1}^{m} \int_{M_{i}^{\mathrm{pre}}}(1-\chi) \psi_{i} d \Omega+\int_{D^{\text {und }}}(1-\chi) \prod_{i=1}^{m} \psi_{i} d \Omega .
$$




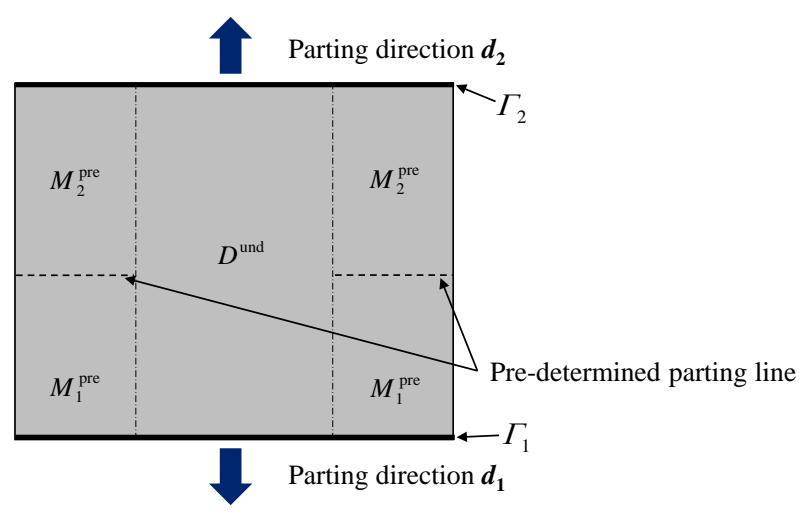

Fig. 8 Predetermination of a parting line.

\section{Topology optimization}

\subsection{Formulation}

In the present study, we focus on topology optimization, which offers the most potential for exploring superior structures among the structural optimization methods. The key idea of topology optimization is the replacement of a structural optimization problem with a material distribution problem by introducing a fixed design domain and the characteristic function that assumes values of 1 or 0 in the fixed design domain. Let $\Omega$ and $D$ denote the material domain and fixed design domain, respectively. The characteristic function $\chi_{\Omega} \in L^{\infty}(D)$ is then defined as

$\chi_{\Omega}(\boldsymbol{x})= \begin{cases}1 & \text { for } \boldsymbol{x} \in \Omega \\ 0 & \text { for } \boldsymbol{x} \in D \backslash \Omega,\end{cases}$

where complementary domain $D \backslash \Omega$ represents a void domain. This characteristic function $\chi_{\Omega}$ is the same as that introduced in Section 2.2 as $\chi$. Since the characteristic function can be very discontinuous, some relaxation or regularization technique is usually used, and the homogenization design method [15] is a representative approach for relaxing the design domain.

On the other hand, level set-based shape optimization methods [21-23] that regularize the design space have been proposed. In these methods, structural boundaries are clearly expressed by the iso-surface of a scaler function, namely, the level set function. However, these methods do not allow the topological changes that generate new boundaries during the optimization procedure, since the level set function in conventional level set-based optimization methods is updated using the Hamilton-Jacobi equation, which requires that the property of the function as a signed distance function be maintained. To allow the creation of the new boundaries, Allaire et al. [42] incorporated the bubble method [43] with a level set-based structural optimization method. On

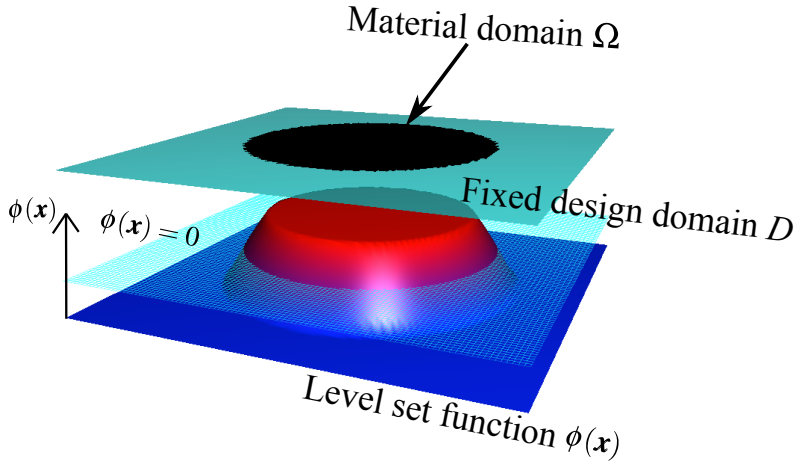

Fig. 9 Level set-based structural representation.

the other hand, Yamada et al. proposed a level set-based topology optimization method $[40,44]$ in which the level set function is defined as a piecewise constant function and is updated using a reaction-diffusion equation. This method allows topological changes that generate new boundaries to occur during the optimization and is the method adopted in the present study, as described below.

Using the level set function $\phi$, structural boundaries are implicitly represented by the iso-surface of the level set function as follows (see Fig. 9):

$$
\begin{cases}1 \geq \phi(x)>0 & \text { for } x \in \Omega \backslash \partial \Omega \\ \phi(x)=0 & \text { for } x \in \partial \Omega \\ 0>\phi(x) \geq-1 & \text { for } x \in D \backslash \Omega .\end{cases}
$$

Based on this structural representation, the characteristic function $\chi_{\Omega}$ is redefined as $\chi_{\phi}$, using the level set function as follows:

$\chi_{\phi}= \begin{cases}1 & \text { for } \phi \geq 0 \\ 0 & \text { for } \phi<0 .\end{cases}$

Let $f_{\mathrm{d}}$ and $f_{\mathrm{b}}$ denote the integrands of an objective functional, the former defined in the fixed design domain $D$ and the latter defined on $\Gamma$, the boundary of $D$. Then, an optimization problem to minimize an objective functional, $F$, under an inequality constraint, $G$, is formulated as

$$
\inf _{\chi_{\phi}} F\left[\chi_{\phi}\right]=\int_{D} f_{\mathrm{d}}\left(\boldsymbol{x}, \chi_{\phi}\right) d \Omega+\int_{\Gamma} f_{\mathrm{b}}\left(\boldsymbol{x}, \chi_{\phi}\right) d \Gamma
$$

subject to $G\left[\chi_{\phi}\right]=\int_{D} g\left(\boldsymbol{x}, \chi_{\phi}\right) d \Omega-G_{\max } \leq 0$,

where $g$ represents the integrand of the constraint functional and $G_{\max }$ represents the upper limit of the constraint functional. The above optimization problem is ill-posed [45], so some types of relaxation or regularization are required.

\subsection{Level set function updating scheme}

We denote a fictitious time, $t$, and assume that the level set function $\phi$ is implicitly a function of $t$. Using the scheme de- 
veloped in previous research [40, 44], the level set function is updated using the following time evolution equation:

$\frac{\partial \phi}{\partial t}=-K\left(J^{\prime}-\tau \nabla^{2} \phi\right)$,

where $K>0$ is a coefficient of proportionality and $J^{\prime}$ is the design sensitivity, i.e., the gradient of the Lagrangian. The second term of the right-hand side of the above equation, $\tau \nabla^{2} \phi$, is a diffusion term and it ensures the smoothness of the level set function. The diffusion term regularizes the topology optimization problem and the regularization parameter, $\tau$, affects the degree of geometrical complexity of the optimized configurations. A uniform cross-section surface constraint, also called an extrusion constraint, can easily be imposed by redefining the regularization parameter $\tau$ as an anisotropic second order tensor, $\tau$, and replacing the above reaction-diffusion equation with the following:

$\frac{\partial \phi}{\partial t}=-K\left(J^{\prime}-\operatorname{div}(\tau \nabla \phi)\right)$.

If a component of the tensor in the constraint direction is set to a large value, the level set function will remain constant in the constraint direction, due to the large diffusion. Consequently, the obtained optimal configurations will reflect the imposition of a uniform cross-section surface constraint, which may improve manufacturability.

\subsection{Minimum mean compliance problem}

We first formulate a minimum mean compliance problem considering casting or injection molding manufacturability, using the fictitious physical models presented in Section 2.3.

Consider an elastic structure occupying a material domain $\Omega$ that is fixed at boundary $\Gamma_{\mathrm{u}}$, with a traction $t$ applied at $\Gamma_{\mathrm{t}}$. Let $\boldsymbol{u}_{1}, \boldsymbol{C}$ and $\boldsymbol{\varepsilon}$ respectively denote the displacement field, the elastic tensor, and the strain tensor, represented as $\boldsymbol{\varepsilon}\left(\boldsymbol{u}_{1}\right)=\frac{1}{2}\left(\nabla \boldsymbol{u}_{1}+\nabla \boldsymbol{u}_{1}^{\mathrm{T}}\right)$. Using the level set-based structural representation expressed in Eqs. (19) and (20), the optimization problem to determine an optimal configuration of the material domain $\Omega$ that has a minimum mean compliance and appropriate manufacturability features under a volume constraint can be formulated as follows:

$\inf _{\chi_{\phi}} F=\int_{\Gamma_{t}} \boldsymbol{t} \cdot \boldsymbol{u}_{1} d \Gamma+\bar{\gamma} F_{\mathrm{M}}^{m}$

subject to:

$$
\begin{aligned}
G_{1}= & \int_{D} \chi_{\phi} \mathrm{d} \Omega-V_{\max } \leq 0 \\
E_{1}= & \int_{\Gamma_{t}} \boldsymbol{t} \cdot \tilde{\boldsymbol{u}} d \Gamma-\int_{D} \boldsymbol{\varepsilon}\left(\boldsymbol{u}_{1}\right): \boldsymbol{C} \chi_{\phi}: \boldsymbol{\varepsilon}(\tilde{\boldsymbol{u}}) d \Omega=0 \\
E_{i}^{\mathrm{FPM}}= & \int_{D} \beta \chi_{\phi}\left(1-\psi_{i}\right) \tilde{\psi}_{i} d \Omega-\int_{D} L_{i}^{2} \nabla \tilde{\psi}_{i} \cdot \boldsymbol{A}_{i} \nabla \psi_{i} d \Omega \\
& -\int_{D} \tilde{\psi}_{i} L_{i} \boldsymbol{V}_{i} \cdot \nabla \psi_{i} d \Omega=0,
\end{aligned}
$$

for all variables $\left(\boldsymbol{u}_{1}, \tilde{\boldsymbol{u}}\right) \in \mathscr{U} \times \mathscr{U}$ and $\left(\psi_{i}, \tilde{\psi}_{i}\right) \in \mathscr{T}^{i} \times \mathscr{T}^{i}$, where

$$
\begin{aligned}
\mathscr{U} & =\left\{\tilde{\boldsymbol{u}} \in H^{1}(D)^{N}: \tilde{\boldsymbol{u}}=\mathbf{0} \text { on } \Gamma_{\mathrm{u}}\right\}, \\
\mathscr{T}^{i} & =\left\{\tilde{\psi} \in H^{1}(D): \tilde{\psi}=0 \text { on } \Gamma_{i}\right\},
\end{aligned}
$$

for all indices $i=1,2, \ldots, m$, where $m$ is the number of molds and $N$ is the number of spatial dimensions. In the above formulation, the first term of the objective functional represents the mean compliance and the second term is the objective functional expressing the molding constraint. $\bar{\gamma}$ is a weighting coefficient applied to the molding constraint. $G_{1}$ represents the volume constraint and $V_{\max }$ is the upper limit of the allowable material volume. $E_{1}$ is a weak form equilibrium equation for the displacement field, $\boldsymbol{u}_{1} . E_{i}^{\mathrm{FPM}}$ for $i=$ $1,2, \ldots, m$ are the weak forms of the governing equations of the fictitious physical models formulated in Eqs. (11) - (13). $\tilde{\boldsymbol{u}}$ and $\tilde{\psi}_{i}$ are the test function of the weak forms of Eqs. (27) and (28), and $H^{1}(D)$ is a Sobolev space. The above optimization problem is converted into a non-constrained problem using Lagrange's method for undetermined multipliers.

\subsection{Optimum design problem for a compliant mechanism}

Next, we formulate an optimum design problem for a compliant mechanism $[46,47]$ considering casting or injection molding manufacturability, using the fictitious physical models described in Section 2.3.

Consider a material domain $\Omega$, fixed at boundary $\Gamma_{\mathrm{u}}$, with a traction $\boldsymbol{t}_{\text {in }}$ applied at $\Gamma_{\text {in }}$. Let $\boldsymbol{u}_{2}$ denote the displacement field. By introducing a dummy traction, $\boldsymbol{t}_{\text {out }}$, representing the direction of the specified deformation at output port $\Gamma_{\text {out }}$, an optimum design problem for a compliant mechanism can be formulated as the following mutual mean compliance maximization problem:

$\inf _{\chi_{\phi}} F=-\int_{\Gamma_{\text {out }}} \boldsymbol{t}_{\text {out }} \cdot \boldsymbol{u}_{2} d \Gamma+\bar{\gamma} F_{\mathrm{M}}^{m}$

subject to:

$$
\begin{aligned}
G_{1}= & \int_{D} \chi_{\phi} \mathrm{d} \Omega-V_{\max } \leq 0 \\
E_{2}= & \int_{\Gamma_{\mathrm{out}}} \boldsymbol{t}_{\mathrm{out}} \cdot \tilde{\boldsymbol{u}} d \Gamma-\int_{\Gamma_{\mathrm{out}}} k_{\mathrm{out}} \boldsymbol{u}_{2} \cdot \tilde{\boldsymbol{u}} d \Gamma-\int_{\Gamma_{\mathrm{in}}} k_{\mathrm{in}} \boldsymbol{u}_{2} \cdot \tilde{\boldsymbol{u}} d \Gamma \\
& -\int_{D} \boldsymbol{\varepsilon}\left(\boldsymbol{u}_{2}\right): \boldsymbol{C} \chi_{\phi}: \boldsymbol{\varepsilon}(\tilde{\boldsymbol{u}}) d \Omega=0 \\
E_{i}^{\mathrm{FPM}}= & \int_{D} \beta \chi_{\phi}\left(1-\psi_{i}\right) \tilde{\psi}_{i} d \Omega-\int_{D} L_{i}^{2} \nabla \tilde{\psi}_{i} \cdot \boldsymbol{A}_{i} \nabla \psi_{i} d \Omega \\
& -\int_{D} \tilde{\psi}_{i} L_{i} \boldsymbol{V}_{i} \cdot \nabla \psi_{i} d \Omega=0
\end{aligned}
$$

for all variables $\left(\boldsymbol{u}_{2}, \tilde{\boldsymbol{u}}\right) \in \mathscr{U} \times \mathscr{U}$ and $\left(\psi_{i}, \tilde{\psi}_{i}\right) \in \mathscr{T}^{i} \times \mathscr{T}^{i}$ and all indices $i=1,2, \ldots, m$. In the above formulation, the first term of the objective functional represents the mutual 
mean compliance and the second term is the objective functional that imposes the molding constraint. $G_{1}$ represents the volume constraint and $V_{\max }$ is the upper limit of the allowable material volume. $E_{2}$ is the weak form equilibrium equation of the displacement field, $\boldsymbol{u}_{2}$. The second and the third terms of $E_{2}$ represent Robin boundary conditions, for which parameters $k_{\text {out }}$ and $k_{\text {in }}$ allow the setting of reaction forces, due to deformation at boundaries $\Gamma_{\text {out }}$ and $\Gamma_{\mathrm{in}}$, that automatically ensure appropriate structural stiffness.

\subsection{Sensitivity analysis}

To derive the design sensitivity, we represent the objective functional as follows:

$F=\int_{\Gamma} f(\boldsymbol{u}) d \Gamma+\bar{\gamma} F_{\mathrm{M}}^{m}$

where $\boldsymbol{u}=\boldsymbol{u}_{1}$ for the minimum mean compliance problem and $\boldsymbol{u}=\boldsymbol{u}_{2}$ for the optimum design problem for a compliant mechanism. The Lagrangian, $J$, for the optimization problem is defined as

$$
\begin{aligned}
J= & F\left[\boldsymbol{v}, \theta_{i}, \chi_{\phi}\right]+\lambda G\left[\chi_{\phi}\right] \\
& +E_{\alpha}\left[\boldsymbol{v}, \tilde{\boldsymbol{v}}, \chi_{\phi}\right]+\bar{\gamma} \sum_{i=1}^{m} E_{i}^{\mathrm{FPM}}\left[\theta_{i}, \tilde{\theta}_{i}, \chi_{\phi}\right],
\end{aligned}
$$

where $v$ and $\theta_{i}$ are the state variables, $\lambda$ is the Lagrange multiplier for the inequality volume constraint, and $\tilde{\boldsymbol{v}}$ and $\tilde{\theta}_{i}$ are the Lagrange multipliers for the governing equations. $\alpha=1$ for the minimum mean compliance problem and $\alpha=2$ for the compliant mechanism design problem. The stationary conditions for Lagrange multipliers $\tilde{\boldsymbol{v}}$ and $\tilde{\theta}_{i}$ require that each Gâteaux derivative of the Lagrangian with respect to the Lagrange multipliers $\tilde{\boldsymbol{v}}$ and $\tilde{\theta}_{i}$ be equal to 0 , as follows:

$d J(\tilde{\boldsymbol{v}} ; \delta \tilde{\boldsymbol{v}})=E_{\alpha}\left[\boldsymbol{v}, \delta \tilde{\boldsymbol{v}}, \chi_{\phi}\right]=0$

$d J\left(\tilde{\theta}_{i} ; \delta \tilde{\theta}_{i}\right)=\bar{\gamma} E_{i}^{\mathrm{FPM}}\left[\theta_{i}, \delta \tilde{\theta}_{i}, \chi_{\phi}\right]=0$,

for all indices $i=1,2, \ldots, m$. The above conditions can be satisfied if the state variables $\boldsymbol{v}$ and $\theta_{i}$ correspond to the solutions of the governing equations $\boldsymbol{u}$ and $\psi_{i}$, respectively. Similarly, the stationary conditions for the state variables $\boldsymbol{v}$ and $\theta_{i}$ require that each Gâteaux derivative of the Lagrangian with respect to the state variables $v$ and $\theta_{i}$ be equal to 0 , as follows:

$$
\begin{aligned}
& d J(\boldsymbol{v} ; \delta \boldsymbol{v})=F\left[\delta \boldsymbol{v}, \theta_{1}, \theta_{2}, \chi_{\phi}\right]+E_{\alpha}\left[\delta \boldsymbol{v}, \tilde{\boldsymbol{v}}, \chi_{\phi}\right]=0 \\
& d J\left(\theta_{i} ; \delta \theta_{i}\right)=F\left[\boldsymbol{v}, \delta \theta_{i}, \chi_{\phi}\right]+\bar{\gamma} E_{i}^{\mathrm{FPM}}\left[\delta \theta_{i}, \tilde{\theta}_{i}, \chi_{\phi}\right]=0,
\end{aligned}
$$

for all indices $i=1,2, \ldots, m$. To satisfy these conditions, we introduce the following equations using the adjoint variable method:

$$
\begin{aligned}
\tilde{E}_{1}= & \int_{\Gamma_{t}} \boldsymbol{t} \cdot \tilde{\boldsymbol{p}} d \Gamma-\int_{D} \boldsymbol{\varepsilon}(\boldsymbol{p}): \boldsymbol{C} \chi_{\phi}: \boldsymbol{\varepsilon}(\tilde{\boldsymbol{p}}) d \Omega=0 \\
\tilde{E}_{2}= & -\int_{\Gamma_{\mathrm{out}}} \boldsymbol{t}_{\mathrm{out}} \cdot \tilde{\boldsymbol{p}} d \Gamma-\int_{\Gamma_{\mathrm{out}}} k_{\mathrm{out}} \boldsymbol{p} \cdot \tilde{\boldsymbol{p}} d \Gamma-\int_{\Gamma_{\mathrm{in}}} k_{\mathrm{in}} \boldsymbol{p} \cdot \tilde{\boldsymbol{p}} d \Gamma \\
& -\int_{D} \boldsymbol{\varepsilon}(\boldsymbol{p}): \boldsymbol{C} \chi_{\phi}: \boldsymbol{\varepsilon}(\tilde{\boldsymbol{p}}) d \Omega=0 \\
\tilde{E}_{i}^{\mathrm{FPM}}= & \int_{D}\left(1-\chi_{\phi}\right) \prod_{j=1, j \neq i}^{m} \psi_{j} \tilde{\xi}_{i} d \Omega-\int_{D} \beta \chi_{\phi} \xi_{i} \tilde{\xi}_{i} d \Omega \\
& -\int_{D} L_{i}^{2} \nabla \tilde{\xi}_{i} \cdot \boldsymbol{A}_{i} \nabla \xi_{i} d \Omega+\int_{D} \tilde{\xi}_{i} L_{i} \boldsymbol{V}_{i} \cdot \nabla \xi_{i} d \Omega \\
& -\int_{\partial D \backslash\left(\Gamma_{i}\right)} L_{i} \boldsymbol{V}_{i} \cdot \boldsymbol{n} \xi_{i} \tilde{\xi}_{i} d \Gamma \\
= & 0,
\end{aligned}
$$

for all variables $(\boldsymbol{p}, \tilde{\boldsymbol{p}}) \in \mathscr{U} \times \mathscr{U}$ and $\left(\xi_{i}, \tilde{\xi}_{i}\right) \in \mathscr{T}^{i} \times \mathscr{T}^{i}$, and all indices $i=1,2, \ldots, m$. In the above formulation, $\tilde{E}_{1}$ represents the adjoint equation for the minimum mean compliance problem and $\tilde{E}_{2}$ represents the adjoint equation for the compliant mechanism optimum design problem. Note that since $\tilde{E}_{1}$ is identical to $E_{1}$, due to the minimum mean compliance problem being self-adjoint, we can easily obtain that $\boldsymbol{p}=\boldsymbol{u}_{1} . \tilde{E}_{i}^{\mathrm{FPM}}$ for $i=1,2, \ldots, m$ represent the weak forms of the adjoint equations for the variables, $\psi_{i}$, and $\tilde{\boldsymbol{p}}$ and $\tilde{\xi}_{i}$ are the test functions of the weak forms of Eqs. (41)-(43). Substituting these adjoint variables for the $\tilde{\boldsymbol{v}}$ and $\tilde{\theta}_{i}$ in the condition formulations (39) and (40) satisfies the stationary conditions. Finally, the design sensitivity can be derived as follows:

$$
\begin{aligned}
J^{\prime}= & F\left[\boldsymbol{u}, \psi_{i}, \delta \chi_{\phi}\right]+\lambda G\left[\delta \chi_{\phi}\right] \\
& +E_{\alpha}\left[\boldsymbol{u}, \boldsymbol{p}, \delta \chi_{\phi}\right]+\bar{\gamma} \prod_{i=1}^{m} E_{i}^{\mathrm{FPM}}\left[\psi_{i}, \xi_{i}, \delta \chi_{\phi}\right] \\
= & F_{\mathrm{C}}^{\prime}+\bar{\gamma} F_{\mathrm{M}}^{\prime}+\lambda,
\end{aligned}
$$

where

$$
\begin{aligned}
F_{\mathrm{C}}^{\prime} & =-\boldsymbol{\varepsilon}(\boldsymbol{p}): \boldsymbol{C}: \boldsymbol{\varepsilon}(\boldsymbol{u}), \\
F_{\mathrm{M}}^{\prime} & =\sum_{i=1}^{m} \beta\left(1-\psi_{i}\right) \xi_{i}-\prod_{i=1}^{m} \psi_{i} .
\end{aligned}
$$

The level set function is updated using Eqs. (23) and (44). The details of the optimization algorithm are described next.

\section{Numerical implementation}

\subsection{Optimization algorithm}

In the optimization algorithm, the initial level set function is set in the first step and the governing equations are solved using the finite element method (FEM) in the second step. In the third step, the objective functional is evaluated using 


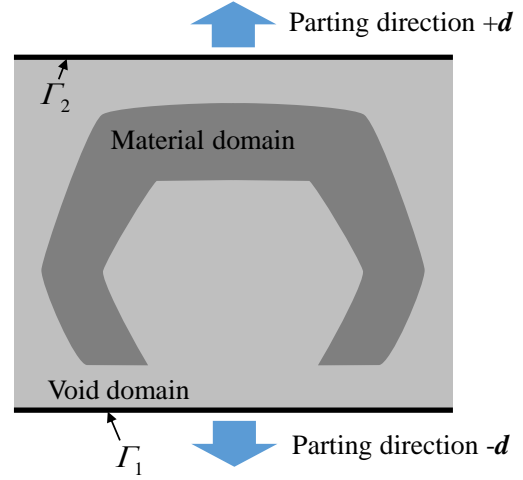

(a)

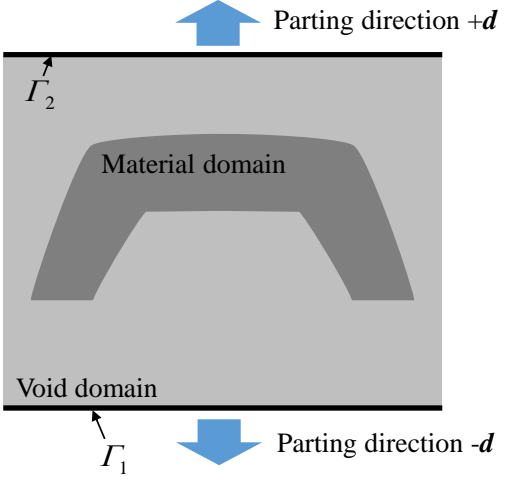

(b)

Fig. 10 Evaluation models: (a) with undercut (Case 1); (b) manufacturable (Case 2).

Eqs. (25) or (31). If the objective functional is converged, the optimization procedure is halted, otherwise the adjoint equations are solved using the FEM. In the fourth step, the sensitivities are evaluated. In the fifth step, the level set function is updated using the reaction-diffusion equation and the optimization procedure then returns to the second step.

\subsection{Approximated characteristic function}

In the present study, the characteristic function $\chi_{\phi}$ is replaced with a smoothed Heaviside function $H_{\phi}$, since $\chi_{\phi}$ is discontinuous and numerical oscillations occur during the optimization procedure, as follows:

$H_{\phi}= \begin{cases}1 & \text { for } \phi>w \\ \frac{1}{2}+\frac{\phi}{w}\left(\frac{15}{16}-\frac{\phi^{2}}{w^{2}}\left(\frac{5}{8}-\frac{3}{16} \frac{\phi^{2}}{w^{2}}\right)\right) & \text { for }-w \leq \phi \leq w \\ 0 & \text { for } \phi<-w\end{cases}$

where $w$ represents the width of the transition.

Furthermore, we use the ersatz material approach [23]. That is, the equilibrium equations (27) and (33) are respectively approximated with the following equations:

$\int_{\Gamma_{t}} \boldsymbol{t} \cdot \tilde{\boldsymbol{u}} d \Gamma-\int_{D} \boldsymbol{\varepsilon}\left(\boldsymbol{u}_{1}\right): \boldsymbol{C}\left(H_{\phi}(1-d)+d\right): \boldsymbol{\varepsilon}(\tilde{\boldsymbol{u}}) d \Omega=0$,

$\int_{\Gamma_{\mathrm{out}}} \boldsymbol{t}_{\mathrm{out}} \cdot \tilde{\boldsymbol{u}} d \Gamma-\int_{\Gamma_{\mathrm{out}}} k_{\mathrm{out}} \boldsymbol{u}_{2} \cdot \tilde{\boldsymbol{u}} d \Gamma-\int_{\Gamma_{\mathrm{in}}} k_{\mathrm{in}} \boldsymbol{u}_{2} \cdot \tilde{\boldsymbol{u}} d \Gamma$
$-\int_{D} \boldsymbol{\varepsilon}\left(\boldsymbol{u}_{2}\right): \boldsymbol{C}\left(H_{\phi}(1-d)+d\right): \boldsymbol{\varepsilon}(\tilde{\boldsymbol{u}}) d \Omega=0$,

where $d$ represents the ratio of the Young's modulus for the two materials, one structural and the other void. Parameter $d$ is introduced to avoid singularities in the stiffness matrix.

\subsection{Normalization for sensitivity scaling}

The scale of the sensitivity $F_{C}^{\prime}$ for the mean compliance or the mutual mean compliance is significantly influenced by the scale of the fixed design domain and the setting of the boundary conditions, whereas the scale of the sensitivity $F_{M}^{\prime}$ for the molding constraint is relatively constant, due to the non-dimensional definition of the fictitious physical models. This makes it difficult to set appropriate values for the weighting coefficient, $\bar{\gamma}$. In the present study, we therefore normalize the sensitivity $F_{\mathrm{C}}^{\prime}$, as follows:

$\overline{F_{\mathrm{C}}^{\prime}}=\frac{F_{\mathrm{C}}^{\prime} \int_{D} d \Omega}{\int_{D}\left|F_{\mathrm{C}}^{\prime}\right| d \Omega}$

Then, we replace the sensitivity $J^{\prime}$ in Eq. (44) with the normalized sensitivity, so that

$\bar{J}^{\prime}=\bar{F}_{\mathrm{C}}^{\prime}+\gamma F_{\mathrm{M}}^{\prime}$,

where $\gamma$ is a weighting coefficient. Consequently, the reactiondiffusion equation for updating the level set function is redefined as

$\frac{\partial \phi}{\partial t}=-K\left(\bar{J}^{\prime}-\tau \nabla^{2} \phi\right)$

This reaction-diffusion equation is discretized in the time direction using the finite difference method and in the spatial direction using the FEM.

\section{Numerical examples}

\subsection{Evaluation of manufacturability}

We first examine the validity of the manufacturability evaluation method using the two fictitious physical models. We consider two structures, Case 1 that has undercut geometry, and Case 2 that has appropriate manufacturability, as 


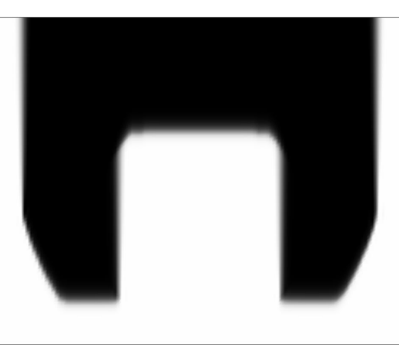

(a)

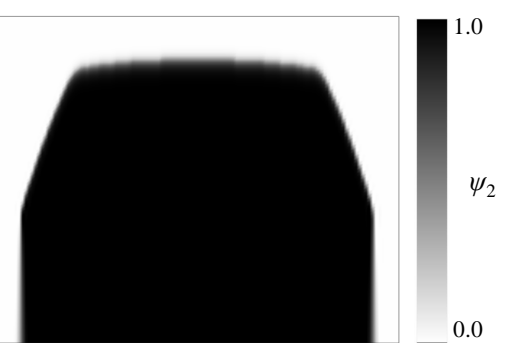

(b)

Fig. 11 Fictitious physical fields: (a) $\psi_{1}$; (b) $\psi_{2}$.

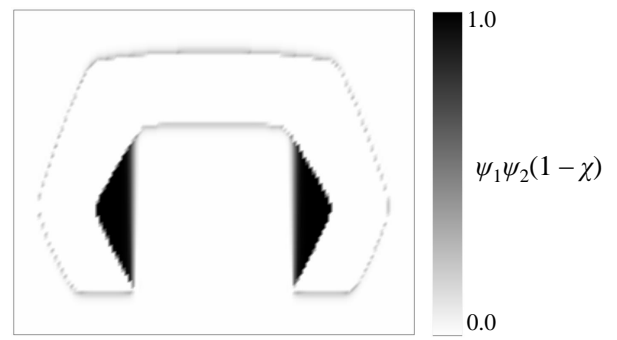

(a)

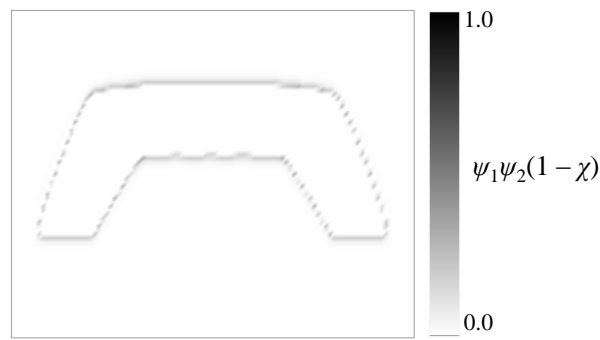

(b)

Fig. 12 The distribution of $\psi_{1} \psi_{2}(1-\chi)$ for the structures: (a) with undercut (Case 1); (b) that can be manufactured (Case 2).

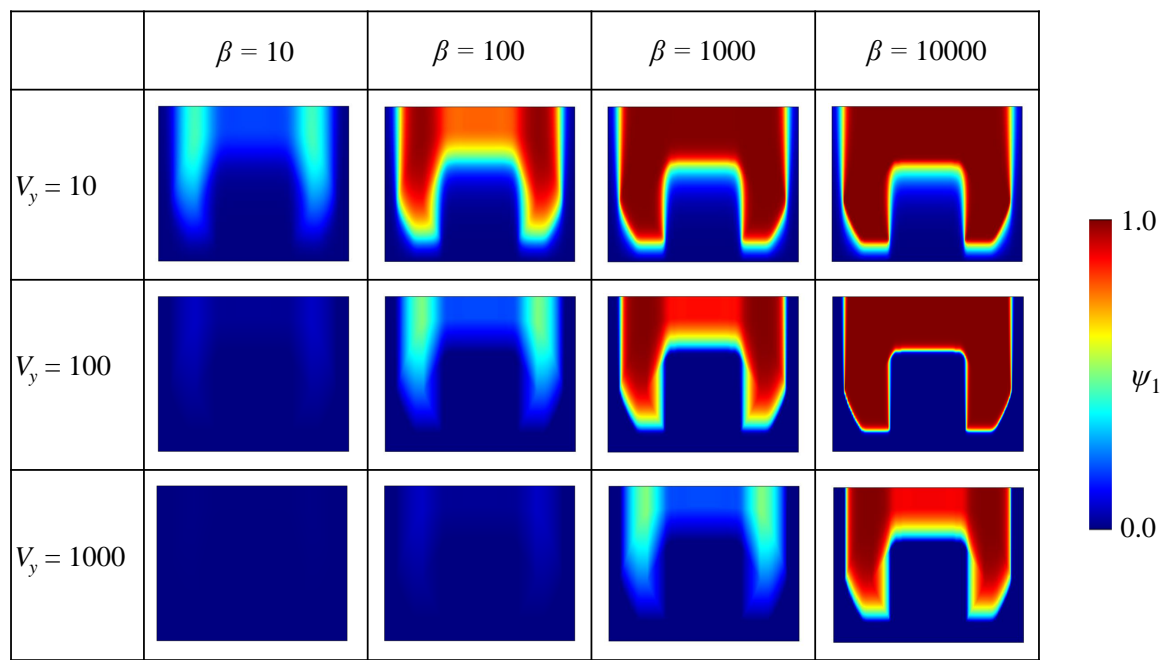

Fig. 13 Fictitious physical field $\psi_{1}$.

respectively shown in Figs. 10(a) and (b). We evaluate the manufacturability of these structures when a two-part mold is used and the parting direction is aligned with the vertical axis. Values for the diffusion tensor $\boldsymbol{A}$ and advection vector $\boldsymbol{V}$ of the fictitious physical models were set as follows:

$\boldsymbol{A}=\left[\begin{array}{ll}\varepsilon & 0 \\ 0 & 1\end{array}\right], \boldsymbol{V}=\left[\begin{array}{c}0 \\ V_{y}\end{array}\right]$,

where $\varepsilon=10^{-2}$ and $V_{y}=100$. Parameter $\beta$ in Eqs. (1) and (4) was set to $10^{4}$. Figures 11 (a) and (b) show the fictitious physical fields $\psi_{1}$ and $\psi_{2}$ obtained by solving Eqs. (1) - (6), and Fig. 12(a) shows the distribution of $\psi_{1} \psi_{2}(1-\chi)$ for Case 1. Figure 12(b) shows the distribution of $\psi_{1} \psi_{2}(1-\chi)$ for Case 2. As shown, regions with high values of $\psi_{1} \psi_{2}(1-$ $\chi)$ correspond to shadow regions, indicating a geometry that would be problematic to manufacture. We can confirm that such regions are successfully highlighted using the proposed fictitious physical models incorporating advection-diffusion equations, indicating that our proposed method can function effectively as a DFM tool. 
5.2 Dependency of $\psi_{1}$ and $\psi_{2}$ on advection velocity and parameter $\beta$ value

To determine appropriate parameter settings, we first examine the dependency of the fictitious physical fields $\psi_{1}$ and $\psi_{2}$ with respect to different settings of the advection velocity $\boldsymbol{V}$ and value for $\beta$, defined in Eqs. (1)-(6). In this example, we use the same model as that shown in Fig. 10(a). That is, we assume a two-part mold with opposed vertical parting directions for the parts. Values for the diffusion tensor $\boldsymbol{A}$ and advection vector $\boldsymbol{V}$ of the fictitious physical models were set as indicated in Eq. (53). To ensure that the values of the fictitious physical fields $\psi_{1}$ and $\psi_{2}$ correctly represent shadow and non-shadow areas, namely that $\psi_{1}$ and $\psi_{2}$ are nearly 0 in "light-irradiated" regions and 1 in shadow regions, appropriate values for $V_{y}$ and $\beta$ must be set. We only deal with $\psi_{1}$ here, since the properties of $\psi_{1}$ and $\psi_{2}$ are very similar. Figure 13 shows fictitious physical field $\psi_{1}$ for various settings of $V_{y}$ and $\beta$, and indicates that $\beta$ should be set to values at least 10 times larger than that of the advection velocity, $V_{y}$.

5.3 Topology optimization for two-dimensional minimum mean compliance problem

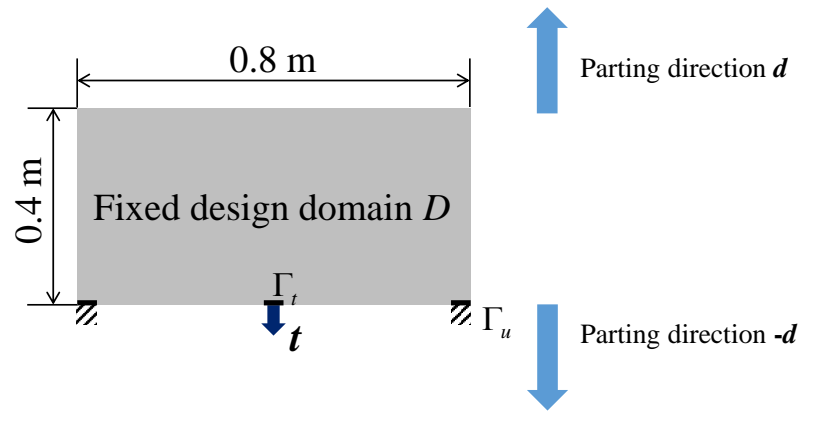

Fig. 14 Fixed design domain and boundary conditions of the twodimensional problem.

We now combine the proposed method with the level set-based topology optimization. First, the proposed method is applied to a two-dimensional minimum mean compliance problem to confirm the validity and utility of the proposed molding constraint. Figure 14 shows the fixed design domain and boundary conditions. The isotropic linearly elastic material employed has Young's modulus $=210 \mathrm{GPa}$ and Poisson's ratio $=0.31$, respectively. The upper limit of the allowable volume was set to $40 \%$ of the volume of the fixed design domain. The regularization parameter $\tau$ was set to $1 \times 10^{-4}$. We assume a two-part mold that parts in opposite directions along the $y$-axis for this two-dimensional problem. In all of the following examples of the two-dimensional problem, the diffusion tensor $\boldsymbol{A}$ and advection vector $\boldsymbol{V}$ of the fictitious physical models were set as indicated in Eq. (53), and parameter $\beta$ was set to $10 V_{y}$. In the following examples, we examine the dependency of the optimal configurations on the value of parameter $\gamma$ and on the initial configuration.

\subsubsection{Dependency of optimal configurations on parameter $\gamma$}

First, we examine the dependency of the optimal configurations with respect to different settings of parameter $\gamma$. In this example, $V_{y}$ was set to 100 and the fixed design domain was filled with material for the initial configuration.

Various obtained optimal configurations are depicted in Fig. 15(a)-(g). The optimal configuration obtained in the absence of a molding constraint is shown in Fig. 15(a), for comparison, and we observe that several interior voids are present, indicating that this configuration cannot be manufactured by casting or injection molding. On the other hand, the optimal configurations in Fig. 15(c)-(g) show geometrical features that are appropriate for manufacturing when the mold parting direction is set along the $y$-axis. However, as shown in Fig. 15(b), when $\gamma$ is set to a relatively small value, the molding constraint fails to prevent the inclusion of void areas in the optimal configuration. When $\gamma$ is set to too large a value, the performance of the obtained optimal configuration (Fig. 15(g)) is considerably below that of the other configurations (Fig. 15(a)-(f)). However, the obtained optimal configurations are almost identical when $\gamma$ is set in the range of $0.5-10$. Consequently, we can confirm that optimal configurations with appropriate manufacturability show little dependency on the value of weighting coefficient $\gamma$.

\subsubsection{Dependency of optimal configurations on the initial configuration}

Next, we examine the dependency of the optimal configurations with respect to different setting of the initial configurations, using three different configurations. The initial configuration for Case 1 has material filling the fixed design domain, a configuration of eight holes for Case 2, and material filling only the lower half of the fixed design domain for Case 3. In this example, $\gamma$ and $V_{y}$ were set to 1 and 100 , respectively.

Initial, intermediate, and obtained optimal configurations are shown in Fig. 16. The obtained optimal configuration are almost identical in these three cases, so we confirm that the dependency of the optimal configurations on the initial configuration is extremely low. Furthermore, the results shown in Fig. 16 demonstrate that intermediate results during the optimization procedure can violate the molding constraint although it is satisfied in the final optimal configurations. This advantageous behavior occurs because the proposed method does not restrict the design space, but imposes the molding constraint via the fictitious physical models. 


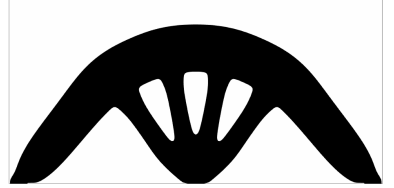

(a)

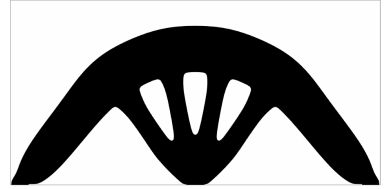

(b)

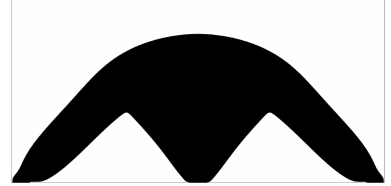

(c)

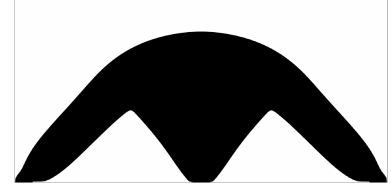

(d)

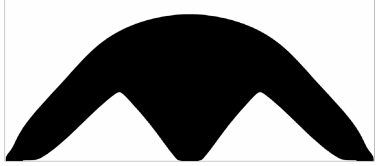

(e)

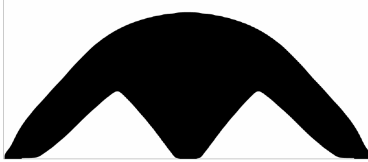

(f)

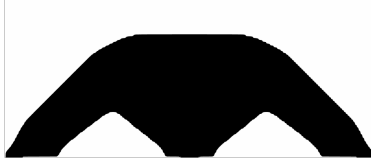

(g)

Fig. 15 Optimal configurations: (a) without molding constraint, mean compliance $=2.90 \times 10^{-8} \mathrm{~J}$; (b) $\gamma=0.1$, mean compliance $=2.91 \times 10^{-8}$ $\mathrm{J}$; (c) $\gamma=0.5$, mean compliance $=2.97 \times 10^{-8} \mathrm{~J}$; (d) $\gamma=1.0$, mean compliance $=2.97 \times 10^{-8} \mathrm{~J}$; (e) $\gamma=5.0$, mean compliance $=2.97 \times 10^{-8} \mathrm{~J}$; (f) $\gamma=10$, mean compliance $=2.97 \times 10^{-8} \mathrm{~J}$; (g) $\gamma=50$, mean compliance $=3.17 \times 10^{-8} \mathrm{~J}$.

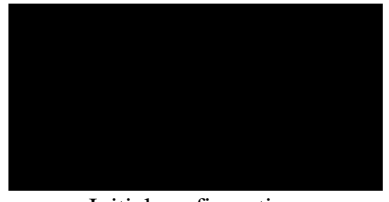

Initial configuration

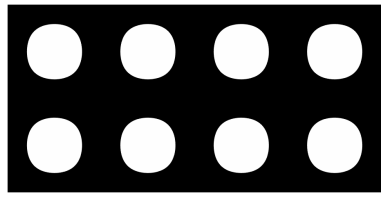

Initial configuration

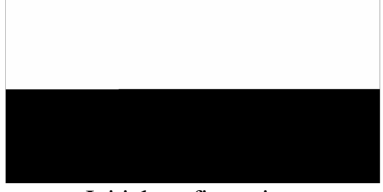

Initial configuration

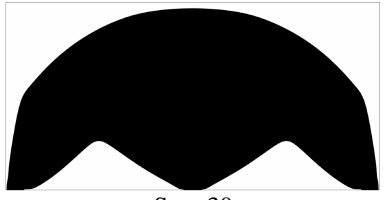

Step 30

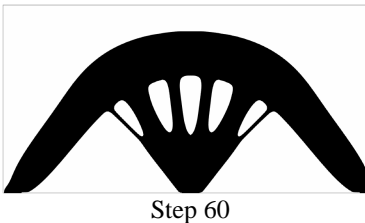

(a)

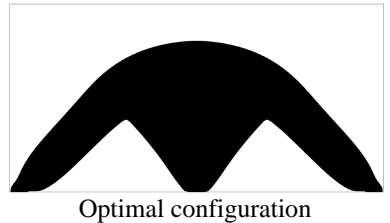

Optimal configuration

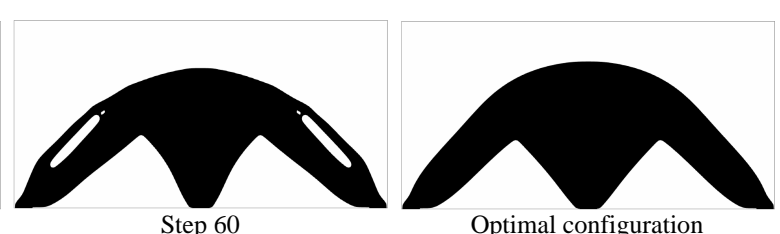

(b)
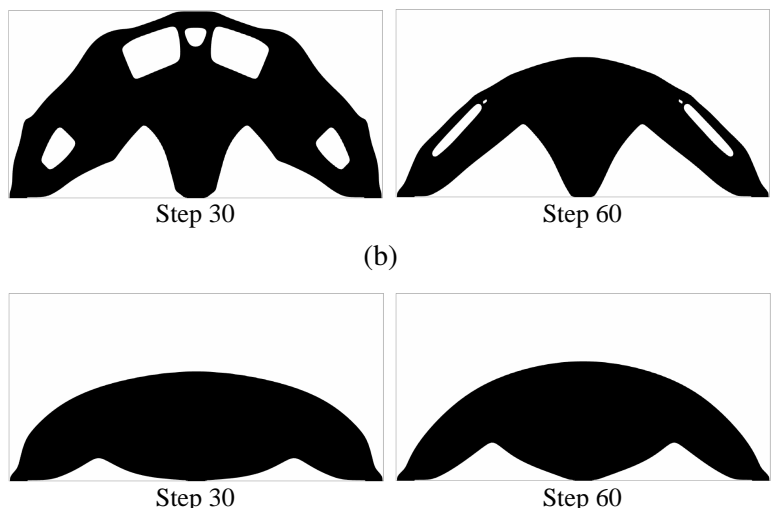

(c)

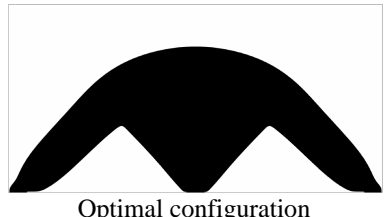

Optimal configuration

Fig. 16 Initial configurations, intermediate results and optimal configurations: (a) Case 1; (b) Case 2; (c) Case 3.

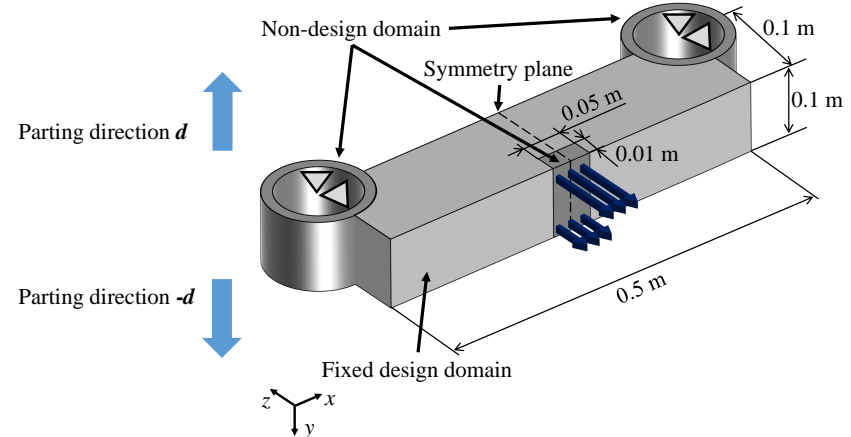

Fig. 17 Fixed design domain and boundary conditions of the threedimensional minimum mean compliance problem.
5.4 Topology optimization for three-dimensional minimum mean compliance problem

We now apply the proposed method to a three-dimensional minimum mean compliance problem. Figure 17 shows the fixed design domain and the boundary conditions. Since the design model is symmetrical, the fixed design domain is defined as only the left half of the illustrated design domain. The inner surfaces of the cylindrical shapes are fixed and the applied traction $\boldsymbol{t}$ is described as follows:

$\boldsymbol{t}= \begin{cases}1000 \mathrm{~N} & 0 \leq y \leq 0.25 \\ 500 \mathrm{~N} & 0.75 \leq y \leq 1.0 \\ 0 \mathrm{~N} & \text { otherwise }\end{cases}$ 

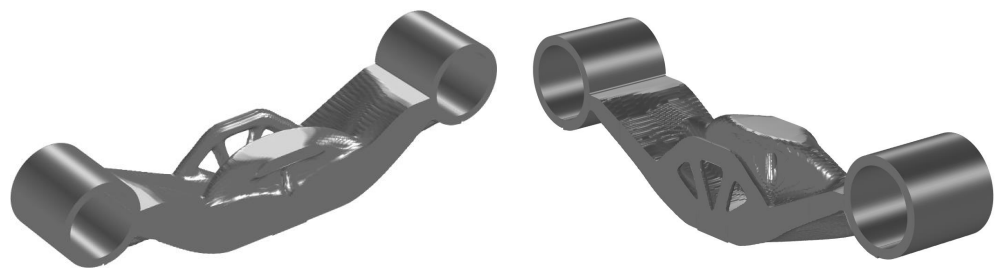

(a)
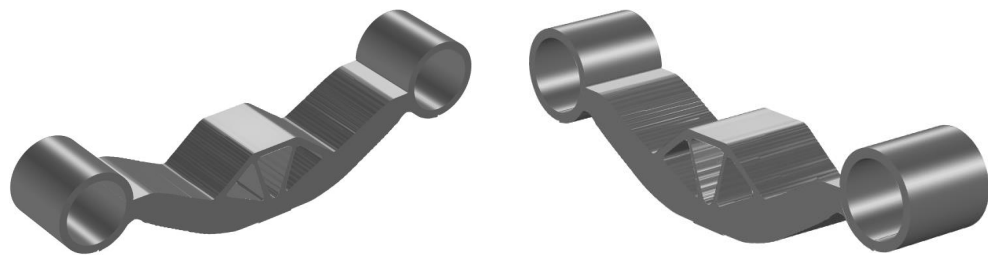

(b)
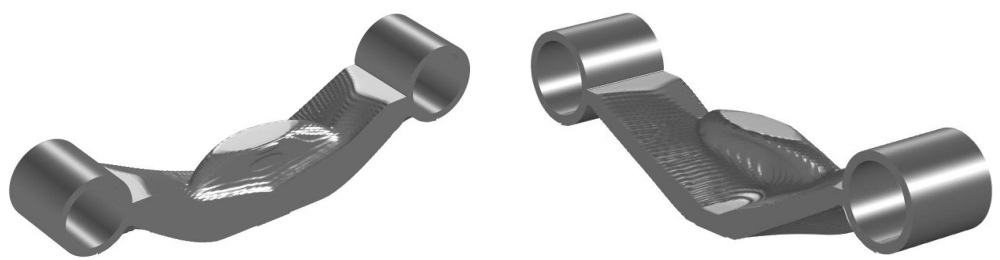

(c)

Fig. 18 Optimal configurations for the three-dimensional minimum mean compliance problem: (a) non-geometrical constraint, mean compliance $=8.05 \times 10^{-10} \mathrm{~J}$; (b) uniform cross-section surface constraint, mean compliance $=8.64 \times 10^{-10} \mathrm{~J}$; (c) proposed molding constraint imposed, mean compliance $=8.19 \times 10^{-10} \mathrm{~J}$.
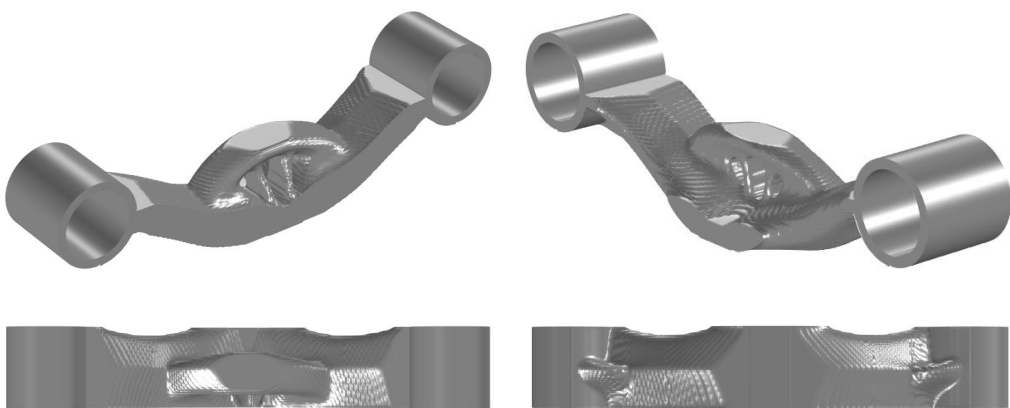

Fig. 19 Optimal configuration for the three-dimensional minimum mean compliance problem with predetermined mold parting line, mean compliance $=8.37 \times 10^{-10} \mathrm{~J}$.

where $y=0$ at the upper surface of the design model. The isotropic linearly elastic material has Young's modulus = $210 \mathrm{GPa}$ and Poisson's ratio $=0.31$, respectively. The upper limit of the allowable volume was set to $40 \%$ of the volume of the fixed design domain. We again assume the use of a two-part mold with parting directions opposed along the $y$-axis. In this problem, to demonstrate the effectiveness of the proposed method, we compare the optimal configurations obtained without a geometrical constraint, when using a uniform cross-section surface constraint, and when using the molding constraint. The regularization parameter $\tau$ was set to $1 \times 10^{-4}$ except for the case of the uniform crosssection surface constraint, where the $x$ and $z$ components of $\tau$ in Eq. (24) were both set to $1 \times 10^{-4}$ and the $y$ component of $\tau$ was set to $1 \times 10^{-1}$. In the fictitious physical models defined in Eqs. (1)-(6), diffusion tensor $\boldsymbol{A}$ and advection vector $V$ of the fictitious physical models were set as follows:

$\boldsymbol{A}=\left[\begin{array}{lll}\varepsilon & 0 & 0 \\ 0 & 1 & 0 \\ 0 & 0 & \varepsilon\end{array}\right], \boldsymbol{V}=\left[\begin{array}{c}0 \\ V_{y} \\ 0\end{array}\right]$,

where $\varepsilon=10^{-2}$. Parameters $\gamma$ and $V_{y}$ were set to 1 and 100 , respectively.

Figure 18 shows the obtained optimal configurations. The optimal solution shown in Fig. 18(a) that was obtained only by focusing on improvement of the physical performance has the smallest mean compliance among these three solutions, although manufacturing this configuration by cast- 

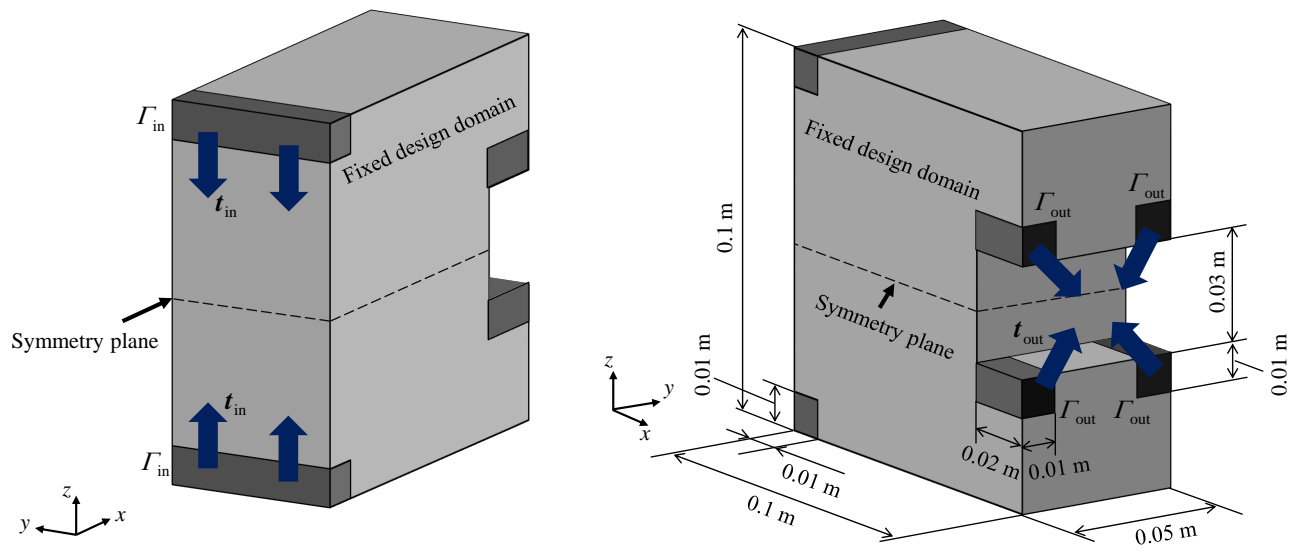

Parting directions

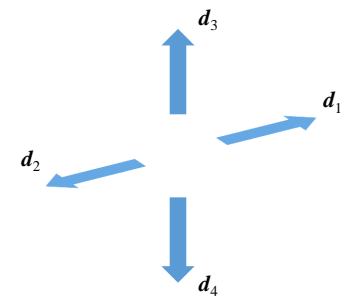

Fig. 20 Fixed design domain and boundary conditions of the optimum design problem for compliant mechanism.
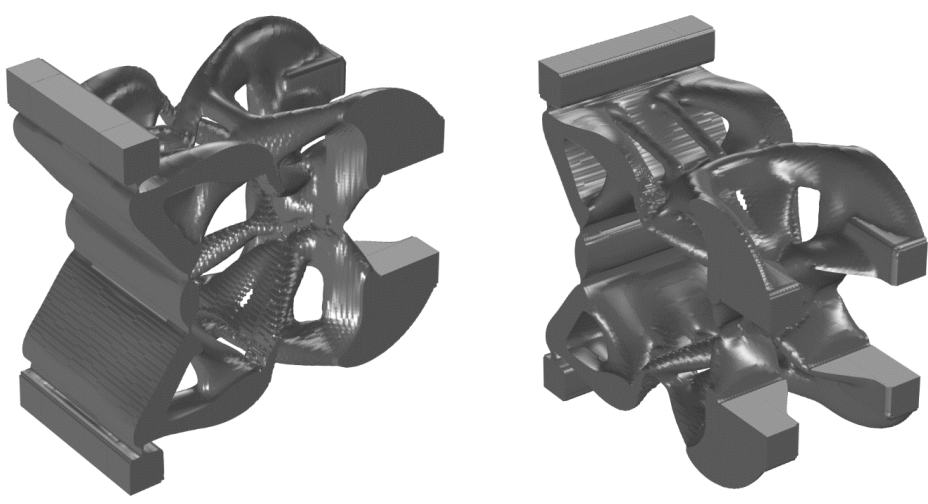

(a)
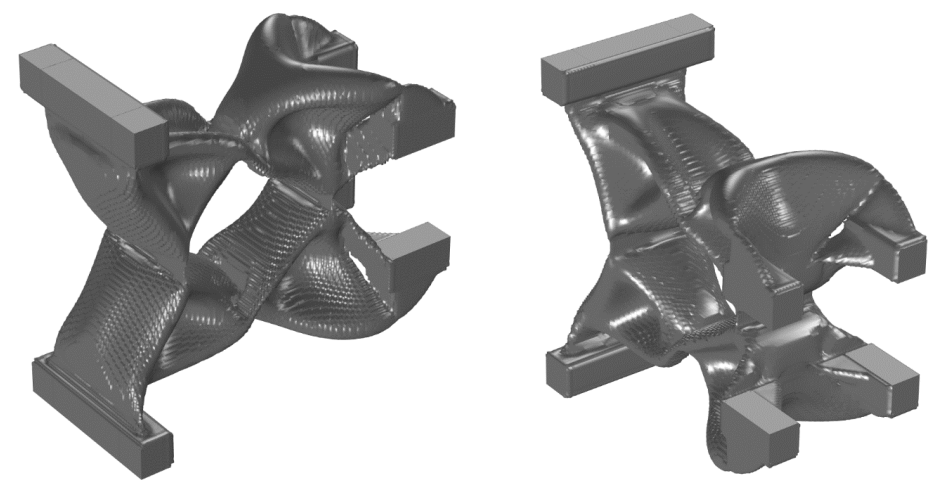

(b)

Fig. 21 Optimal configurations for the optimum design problem of a compliant mechanism obtained (a) without the molding constraint (mutual mean compliance $=2.66 \times 10^{-10} \mathrm{~J}$ ); and (b) with molding constraint imposed (mutual mean compliance $=2.43 \times 10^{-10} \mathrm{~J}$ ).

ing might be difficult since an undercut is included no matter where the parting surface is set. On the other hand, the optimal configuration shown in Fig. 18(b), obtained by imposing a uniform cross-section surface constraint, is simple enough to be manufactured by casting. As is obvious, however, the physical performance, i.e. the mean compliance, of this result is not as good as that of the optimal solution shown in
Fig. 18(a). The optimal configuration shown in Fig. 18(c) also has favorable manufacturability and the mean compliance is lower than that for Fig. 18(b). Therefore, the optimal solution obtained when imposing the molding constraint is a better design candidate when manufacturability is a prerequisite. 


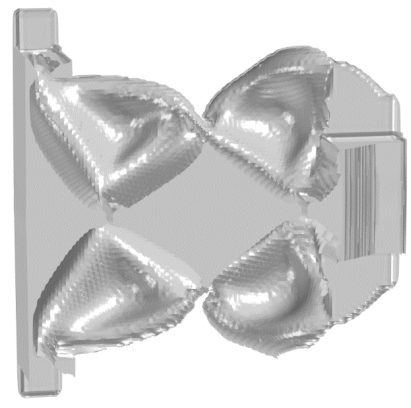

(a)

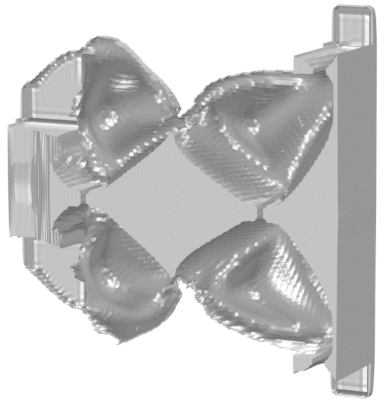

(b)

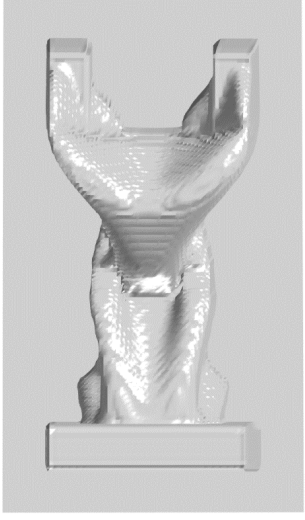

(c)

Fig. 22 Mold parts and parting directions for manufacturing the optimal configuration obtained with molding constraint imposed: (a) $\boldsymbol{d}_{1}$ (Mold part 1); (b) $\boldsymbol{d}_{2}$ (Mold part 2); (c) $\boldsymbol{d}_{3}$ (Mold part 3).

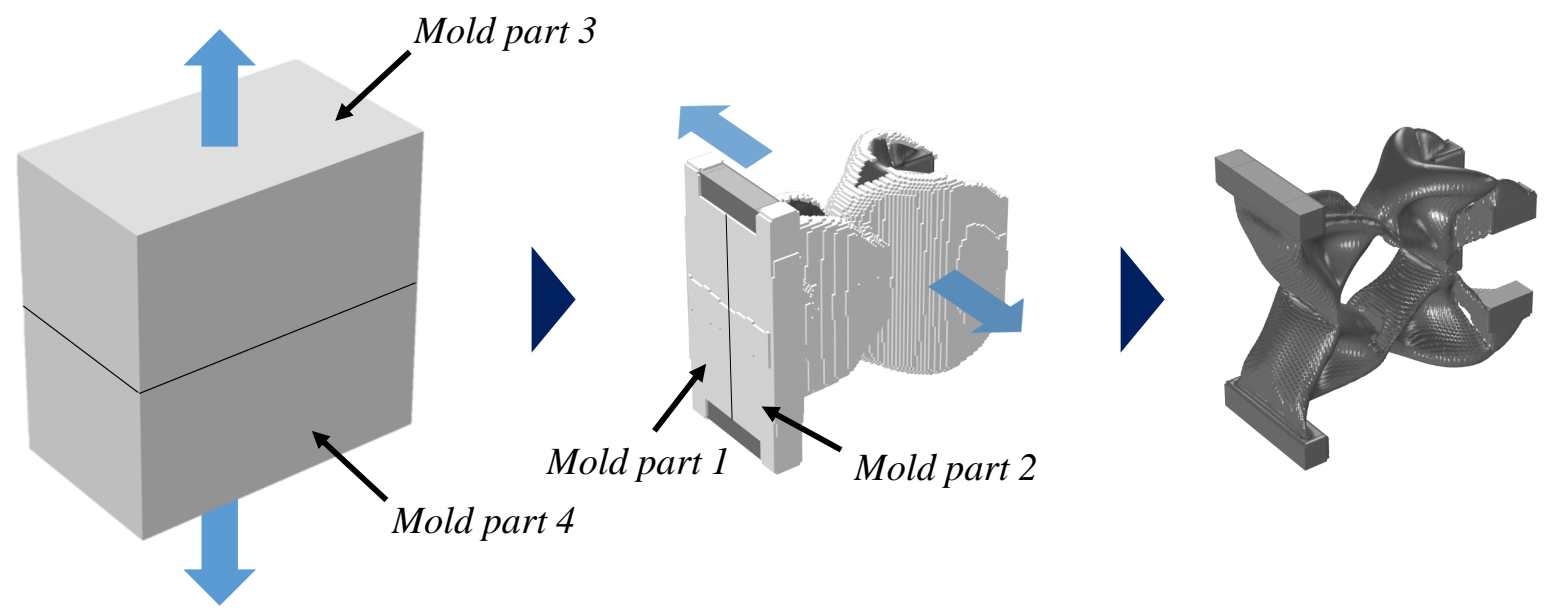

Fig. 23 Sequential separation of molds parts.

\subsubsection{Predetermination of parting line location}

Next, we examine the effectiveness of predetermining the location of the parting line for a two-part mold. We assume a two-part mold with opposed parting directions along the $y$-axis, with the parting line predetermined on the surface $y=0.05$, i.e., at middle of the design domain, based on the formulation in Section 2.4. All parameters are the same as those used in Section 5.4.

Figure 19 shows the optimal configuration. The mean compliance of the optimal configuration here is lower than that of the optimal configuration shown in Fig. 18(b), although it is higher than that of the optimal configuration shown in Fig. 18(c), where the molding constraint is imposed but the parting line is not predetermined. This result indicates that the optimal configuration with the parting line location preset at $y=0.05$ can be manufactured, and that setting the location of the mold parting line prior to the op- timization procedure is a useful alternative to allowing the proposed method to determine the location.

5.5 Topology optimization for an optimum design problem of a compliant mechanism

The proposed method is now applied to an optimum design problem for a compliant mechanism. In this example, the molds for manufacturing the optimized structure will also be designed. Figure 20 shows the fixed design domain and the boundary conditions. Since the design model is symmetrical, the fixed design domain is defined as the upper half of the design domain. A traction, $\boldsymbol{t}_{\mathrm{in}}$, is applied at boundaries $\boldsymbol{t}_{\text {in }}$ that are fixed along the $x$ and $y$ axes. The isotropic linearly elastic material has Young's modulus $=210 \mathrm{GPa}$ and Poisson's ratio $=0.31$, respectively. The upper limit of the allowable volume was set to $20 \%$ of the volume of the 


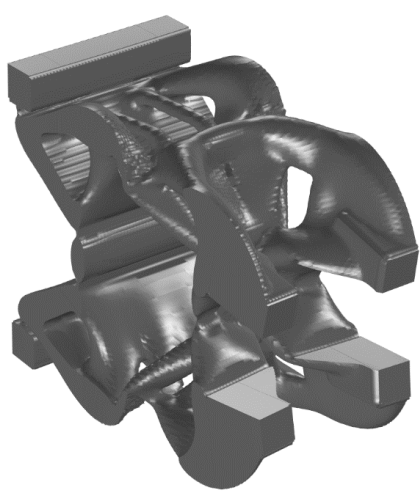

(a)

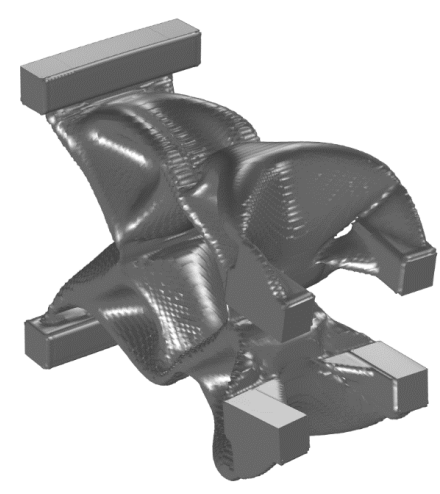

(b)

Fig. 24 Deformed shapes of optimal configurations for the optimum design problem for a compliant mechanism obtained (a) without the molding constraint; (b) with the molding constraint.

fixed design domain. We assume that a four-part mold will be used to manufacture the obtained compliant mechanism design, and let the parting directions be denoted as $\boldsymbol{d}_{1}, \boldsymbol{d}_{2}$, $\boldsymbol{d}_{3}$ and $\boldsymbol{d}_{4}$, as shown in Fig. 20. The regularization parameter $\tau$ was set to $1 \times 10^{-4}$. Due to the symmetry of the design model and the definition of the fixed design domain, we introduce three fictitious physical models that correspond to mold parts with parting directions $\boldsymbol{d}_{1}, \boldsymbol{d}_{2}$, and $\boldsymbol{d}_{3}$. In the fictitious physical models defined in Eqs. (11)-(13), the diffusion tensors $\boldsymbol{A}_{i}$ and advection vectors $\boldsymbol{V}_{i}$ for $i=1,2,3$ of the fictitious physical models were set as follows:

$\boldsymbol{A}_{1}=\boldsymbol{A}_{2}=\left[\begin{array}{lll}\varepsilon & 0 & 0 \\ 0 & 1 & 0 \\ 0 & 0 & \varepsilon\end{array}\right], \boldsymbol{A}_{3}=\left[\begin{array}{lll}\varepsilon & 0 & 0 \\ 0 & \varepsilon & 0 \\ 0 & 0 & 1\end{array}\right]$,

$\boldsymbol{V}_{1}=\left[\begin{array}{c}0 \\ -V_{y} \\ 0\end{array}\right], \boldsymbol{V}_{2}=\left[\begin{array}{c}0 \\ V_{y} \\ 0\end{array}\right], \boldsymbol{V}_{3}=\left[\begin{array}{c}0 \\ 0 \\ V_{z}\end{array}\right]$,

where $\varepsilon=10^{-2}$. Parameter $\gamma$ was set to 5 , and $V_{y}=V_{z}=$ 100.

Figure 21 shows the obtained optimal configurations. The optimal solution shown in Fig. 21(a) obtained when focusing only on improvement of the physical performance has a larger mutual mean compliance than that of the result shown in Fig. 21(b) that was obtained considering the manufacturability. However, the optimal solution obtained when considering the manufacturability has a simpler structure than that obtained without the molding constraint imposed, which facilitates manufacturing by casting.

Figure 22 shows the mold parts for manufacturing the optimal configuration shown in Fig. 21(b). Mold part 4, whose parting direction is $\boldsymbol{d}_{4}$, is identical to that shown in Fig. 22(c) due to the symmetry of the fixed design domain. The shapes of the mold parts are obtained using Eq. (16). As mentioned in Section 2.3, when certain domains intersect, such domains can be included in any relevant mold part. Here, the order in which the mold parts are separated depends on which intersecting domains are included in particular mold parts. In the example shown in Fig. 22, Mold part 3 consists of $M_{3}$, Mold part 1 consists of $\left(M_{1} \cap D_{y^{+}}\right) \backslash M_{3}$, and Mold part 2 consists of $\left(M_{2} \cap D_{y^{-}}\right) \backslash M_{3}$, where $D_{y^{+}}$and $D_{y^{-}}$are defined as follows:

$D_{y^{+}}=\{\forall \boldsymbol{x} \in D: y \geq 0\}$,

$D_{y^{-}}=\{\forall \boldsymbol{x} \in D: y<0\}$,

where $y=0$ at the center of the design model. In the current case, the order in which the mold parts are parted from the cast object is illustrated in Fig. 23. First, Mold part 3 and Mold part 4 are parted and then Mold part 1 and Mold part 2 are parted. That is, Mold part 1 and Mold part 2 operate as casting cores, and the optimal configuration obtained considering the molding constraint is appropriate for manufacture by casting. Note that this mold part design does not include gates, through which molten material enters the mold.

Figure 24 shows the deformed shapes of the two examples of the compliant mechanism three-dimensional design problem. As shown, the optimal configuration obtained when the molding constraint is imposed deforms in the specified direction, confirming the utility of the proposed method.

\section{Conclusion}

This paper presented a method for evaluating the manufacturability of designs when using casting, based on the use of fictitious physical models, and extended it to a scheme for imposing a molding constraint within a topology optimization procedure. We achieved the following:

1. The geometrical requirements for manufacturability using molds were clarified, aiming to facilitate the creation 
of molds in situations where design engineers must ensure that the components do not incorporate undercuts or interior voids.

2. Fictitious physical models were introduced to implicitly represent the manufacturability when using casting or injection molding techniques. The fictitious physical models were mathematically described using advectiondiffusion equations. A molding constraint for use in a structural optimization process was then formulated using the fictitious physical models.

3. In the numerical implementation, an optimization algorithm was constructed and the non-dimensional sensitivity was used to enable simple adjustment of a weighting coefficient.

4. Two- and three-dimensional numerical examples were provided. The dependencies of the distribution of the fictitious physical fields on the setting of advection velocity $\boldsymbol{V}$ and parameter $\beta$ were examined. The proposed method was then applied to topology optimization design problems and obtained manufacturable optimal configurations.

5. In two-dimensional design problems, optimal configurations obtained for each numerical example demonstrated a very low dependency on the setting of the molding constraint weighting coefficient (parameter $\gamma$ ), and the initial configurations.

6. In the minimum mean compliance problem, the proposed method provided an optimal configuration that was manufacturable and had the lowest mean compliance except for the optimal configuration where a molding constraint was not imposed, which could not be manufactured. Furthermore, we demonstrated that the proposed method can set the location of the parting line relative to mold parts prior to the optimization procedure.

7. An optimal design for a compliant mechanism to be manufactured using a four-part mold was obtained. The obtained optimal configuration deformed in the specified direction even though the molding constraint was imposed.

The authors hope to conduct future research to construct a topology optimization method that can consider the entire casting process, including the determination of optimal locations of parting lines and gate layout.

Acknowledgements The authors are grateful to co-researchers in the Mitsubishi Electric Corporation for helpful discussions. The authors also sincerely appreciate the support received from the research scholar of Toyota Physical \& Chemical Research Institute.

\section{References}

1. Xia Q, Shi T, Wang MY, Liu S (2010) A level set based method for the optimization of cast part. Structural and
Multidisciplinary Optimization 41(5):735-747

2. Boothroyd G, Dewhurst P, Knight WA (2002) Product Design for Manufacture and Assembly, Second Edition. CRC press

3. Joshi D, Ravi B (2010) Early castability evaluation using analytical hierarchy process. The International Journal of Advanced Manufacturing Technology 50(1):2136

4. Armillotta A, Fasoli S, Guarinoni A (2016) Cold flow defects in zinc die casting: prevention criteria using simulation and experimental investigations. The International Journal of Advanced Manufacturing Technology 85(1):605-622

5. Hoque A, Halder P, Parvez M, Szecsi T (2013) Integrated manufacturing features and design-formanufacture guidelines for reducing product cost under CAD/CAM environment. Computers \& Industrial Engineering 66(4):988-1003

6. James BD, Spisak AB, Colella WG (2014) Design for Manufacturing and Assembly Cost Estimate Methodology for Transportation Fuel Cell Systems. Journal of Manufacturing Science and Engineering 136(2):024,503

7. Selvaraj P, Radhakrishnan P, Adithan M (2009) An integrated approach to design for manufacturing and assembly based on reduction of product development time and cost. The International Journal of Advanced Manufacturing Technology 42(1-2):13-29

8. Lu C, Zhao WH, Yu SJ (2012) Concurrent tolerance design for manufacture and assembly with a game theoretic approach. The International Journal of Advanced Manufacturing Technology 62(1-4):303-316

9. Liu SG, Jin Q, Wang P, Xie RJ (2014) Closed-form solutions for multi-objective tolerance optimization. The International Journal of Advanced Manufacturing Technology 70(9-12):1859-1866

10. Salonitis K (2016) Design for additive manufacturing based on the axiomatic design method. The International Journal of Advanced Manufacturing Technology 87(1):989-996

11. Prager W (1974) A note on discretized Michell structures. Computer Methods in Applied Mechanics and Engineering 3(3):349-355

12. Svanberg K (1981) Optimization of geometry in truss design. Computer Methods in Applied Mechanics and Engineering 28(1):63-80

13. Pironneau O (1984) Optimal shape design for elliptic systems. Springer-Verlag

14. Sokolowski J, Zolesio JP (1992) Introduction to shape optimization. Springer

15. Bendsøe MP, Kikuchi N (1988) Generating optimal topologies in structural design using a homogenization method. Computer Methods in Applied Mechanics and 
Engineering 71(2):197-224

16. Bendsøe MP (1989) Optimal shape design as a material distribution problem. Structural Optimization 1(4):193202

17. Yaji K, Yamada $\mathrm{T}$, Yoshino $\mathrm{M}$, Matsumoto $\mathrm{T}$, Izui K, Nishiwaki S (2014) Topology optimization using the lattice Boltzmann method incorporating level set boundary expressions. Journal of Computational Physics 274:158-181

18. Otomori M, Yamada T, Izui K, Nishiwaki S, Andkjær J (2012) A topology optimization method based on the level set method for the design of negative permeability dielectric metamaterials. Computer Methods in Applied Mechanics and Engineering 237:192-211

19. Noguchi Y, Yamada T, Otomori M, Izui K, Nishiwaki S (2015) An acoustic metasurface design for wave motion conversion of longitudinal waves to transverse waves using topology optimization. Applied Physics Letters 107(22):221,909

20. Bendsøe MP, Sigmund O (1999) Material interpolation schemes in topology optimization. Archive of Applied Mechanics 69(9-10):635-654

21. Osher S, Sethian JA (1988) Fronts propagating with curvature-dependent speed: algorithms based on Hamilton-Jacobi formulations. Journal of computational physics 79(1):12-49

22. Wang MY, Wang X, Guo D (2003) A level set method for structural topology optimization. Computer Methods in Applied Mechanics and Engineering 192(1):227246

23. Allaire G, Jouve F, Toader AM (2004) Structural optimization using sensitivity analysis and a level-set method. Journal of Computational Physics 194(1):363393

24. Bharanidaran R, Ramesh T (2015) A modified postprocessing technique to design a compliant based microgripper with a plunger using topological optimization. The International Journal of Advanced Manufacturing Technology pp 1-10

25. Liu J, Ma Y (2016) A survey of manufacturing oriented topology optimization methods. Advances in Engineering Software 100:161-175

26. Poulsen TA (2003) A new scheme for imposing a minimum length scale in topology optimization. International Journal for Numerical Methods in Engineering 57(6):741-760

27. Guest JK, Prévost JH, Belytschko T (2004) Achieving minimum length scale in topology optimization using nodal design variables and projection functions. International journal for numerical methods in engineering 61(2):238-254

28. Zhou M, Lazarov BS, Wang F, Sigmund O (2015) Minimum length scale in topology optimization by geomet- ric constraints. Computer Methods in Applied Mechanics and Engineering 293:266-282

29. Guest JK (2009) Imposing maximum length scale in topology optimization. Structural and Multidisciplinary Optimization 37(5):463-473

30. Chen S, Wang MY, Liu AQ (2008) Shape feature control in structural topology optimization. ComputerAided Design 40(9):951-962

31. Guo X, Zhang W, Zhong W (2014) Explicit feature control in structural topology optimization via level set method. Computer Methods in Applied Mechanics and Engineering 272:354-378

32. Allaire G, Jouve F, Michailidis G (2016) Thickness control in structural optimization via a level set method. Structural and Multidisciplinary Optimization 53(6):1349-1382

33. Brackett D, Ashcroft I, Hague R (2011) Topology optimization for additive manufacturing. In: Proceedings of the Solid Freeform Fabrication Symposium, Austin, TX, pp 348-362

34. Leary M, Merli L, Torti F, Mazur M, Brandt M (2014) Optimal topology for additive manufacture: a method for enabling additive manufacture of support-free optimal structures. Materials \& Design 63:678-690

35. Langelaar M (2016) Topology optimization of 3d selfsupporting structures for additive manufacturing. Additive Manufacturing 12:60-70

36. Gaynor AT, Guest JK (2016) Topology optimization considering overhang constraints: Eliminating sacrificial support material in additive manufacturing through design. Structural and Multidisciplinary Optimization pp 1-16

37. Li Q, Chen W, Liu S, Tong L (2016) Structural topology optimization considering connectivity constraint. Structural and Multidisciplinary Optimization

38. Zhou M, Fleury R, Shyy YK, Thomas H, Brennan J (2002) Progress in topology optimization with manufacturing constraints. In: Proceedings of the 9th AIAA MDO conference AIAA-2002-4901

39. Allaire G, Jouve F, Michailidis G (2015) Molding direction constraints in structural optimization via a level-set method

40. Yamada T, Izui K, Nishiwaki S, Takezawa A (2010) A topology optimization method based on the level set method incorporating a fictitious interface energy. Computer Methods in Applied Mechanics and Engineering 199(45-48):2876-2891

41. Li H, Li P, Gao L, Zhang L, Wu T (2015) A level set method for topological shape optimization of $3 \mathrm{~d}$ structures with extrusion constraints. Computer Methods in Applied Mechanics and Engineering 283:615-635

42. Allaire G, De Gournay F, Jouve F, Toader A (2005) Structural optimization using topological and shape 
sensitivity via a level set method. Control and cybernetics 34(1):59

43. Eschenauer HA, Kobelev VV, Schumacher A (1994) Bubble method for topology and shape optimization of structures. Structural optimization 8(1):42-51

44. Otomori M, Yamada T, Izui K, Nishiwaki S (2014) Matlab code for a level set-based topology optimization method using a reaction diffusion equation. Structural and Multidisciplinary Optimization pp 1-14

45. Allaire G (2002) Shape optimization by the homogenization method, vol 146. Springer-Verlag New York

46. Sigmund O (1997) On the design of compliant mechanisms using topology optimization*. Journal of Structural Mechanics 25(4):493-524

47. Nishiwaki S, Frecker MI, Min S, Kikuchi N (1998) Topology optimization of compliant mechanisms using the homogenization method. International Journal for Numerical Methods in Engineering 42:535-559 